\title{
Visuotopic Organization and Extent of V3 and V4 of the Macaque
}

\author{
R. Gattass, A. P. B. Sousa, and C. G. Gross' \\ Instituto de Biofísica da UFRJ, Rio de Janeiro, RJ, Brazil, and 'Department of Psychology, Princeton University, Princeton, \\ New Jersey 08540
}

The representation of the visual field in areas V3 and V4 of the macaque was mapped with multiunit electrodes. Twelve Macaca fascicularis were studied in repeated recording sessions while immobilized and anesthetized.

V3 is a narrow strip (4-5 mm wide) of myeloarchitectonically distinct cortex located immediately anterior to V2. It contains a systematic representation of the central $35-40^{\circ}$ of the contralateral visual field; the representation of the upper quadrant is located ventrally in the hemisphere and that of the lower quadrant, dorsally. There is a small gap between the dorsal (V3d) and ventral (V3v) portions of V3. The representation of the horizontal meridian is adjacent to that in V2 and forms the posterior border of both V3d and V3v. Most or all of the anterior border of V3d consists of the representation of the lower vertical meridian. The entire anterior border of $v 3 v$ consists of the representation of the upper vertical meridian.

V4 is a strip of myeloarchitectonically distinct cortex 5-8 mm wide, immediately anterior to V3. It contains a coarse, but systematic, representation of approximately the central $35-40^{\circ}$ of the contralateral visual field. The representation of the upper visual field is located ventrally in the hemisphere. Most of the representation of the lower visual field is located dorsally. The posterior border of V4 corresponds to the representation of the vertical meridian, and the representation of the horizontal meridian is located at or near its anterior border.

In both V3 and V4, the representation of the central visual field is magnified relative to that of the periphery. In both areas, the size of receptive fields increases with increasing eccentricity; however, at a given eccentricity, the receptive fields of V4 are larger than those of V3.

In primates, more than half of the cerebral cortex is visual in function. This visual cortex consists of over a dozen areas, which are distinguishable on the basis of such criteria as connections, myeloarchitecture, and neuronal response properties (see All-

\footnotetext{
Received Oct. 22, 1986; revised Oct. 22, 1987; accepted Oct. 30, 1987.

This study was carried out when the first two authors were Fogarty Fellows at Princcton University in 1978-1980. We thank S. Gorlick for assistance with the histology, V. P. G. dos Santos for the preparation of the illustrations, L. Ungerleider, T. D. Albright, C. Colby, R. Desimone, and H. Rodman for their comments on the manuscript, and the National Institutes of Health (MH-19420, F05W02855, and F05W02858), the National Science Foundation (BNS 79-05589), the Conselho Nacional de Desenvolvimento Científico e Tecnológico (CNPq, Procs. 30.5654 $76,30.0188 / 80,40.2829 / 84$, and $40.2577 / 85)$, Conselho de Ensino para Graduados e Pesquisa (CEPG), and Financiadora de Estudos e Projetos (FINEP, 41.85.0245.00) for financial assistance.

Correspondence should be addressed to Dr. Gattass, Instituto de Biofisica da UFRJ, Centro de Ciências da Saúde-Bloco G, 21941 Rio de Janeiro, RJ, Brasil. Copyright (C) 1988 Society for Neuroscience $0270-6474 / 88 / 061831-15 \$ 02.00 / 0$
}

man, 1981; Gross et al., 1981; Weller and Kaas, 1981; and Van Essen, 1985). Many of these areas contain a topographically organized representation of the visual field, but in the macaque this organization is known in detail for only a few areas, namely $\mathrm{V} 1$, or striate cortex, V2, and middle temporal (MT) (Daniel and Whitteridge, 1961; Gattass and Gross, 1981; Gattass et al., 1981; Van Essen et al., 1981). In this paper we describe the visuotopic organization of 2 additional visual cortical areas, V3 and V4, as determined by multineuron recordings in chronically implanted, immobilized, and anesthetized macaques.

V3 was first described by Zeki $(1969,1978)$ as a cortical band anterior to $\mathrm{V} 2$, with a representation of the inferior visual field located dorsally and a representation of the superior visual field located ventrally. The cortex anterior to V3, including part of the prelunate gyrus, was named "V4" or "V4 complex" by Zeki and his collaborators (Zeki, 1971; Van Essen and Zeki, 1978). The results reported here are, in part, a confirmation of Zeki's proposals for V3, but present a somewhat different view of the visuotopic organization within V4.

\section{Materials and Methods}

Recording. Twelve Macaca fascicularis, weighing between 3.0 and 4.8 $\mathrm{kg}$, were each recorded on 4-8 occasions. Prior to the first recording session, a recording well and a bolt for holding the head were implanted under anesthesia and aseptic conditions.

The preanesthetic medication, induction, and maintenance of anesthesia, immobilization, monitoring of physiological status, recovery from immobilization, and electrode characteristics havc all been described in detail previously (Gattass and Gross, 1981). Briefly, during recording, the animals were maintained under $70 \%$ nitrous oxide and $30 \%$ oxygen and immobilized with pancuronium bromide. Tungsten microelectrodes with an impedance of about $0.7 \mathrm{M} \Omega$ were used. They usually recorded the activity of several neurons (multiunits) simultaneously.

In each monkey, 26-43 vertical penetrations were made over a 4-week period. The penetrations were placed approximately $1-1.5 \mathrm{~mm}$ apart, forming a grid extending throughout prestriate cortex and adjacent areas. Along each penetration, recording sites were separated by a minimum of $300 \mu \mathrm{m}$.

Visual stimuli. The details of the treatment of the eyes and of the visual stimuli used have been previously described (Gattass and Gross, 1981). Briefly, white, colored, and black stimuli were presented on a translucent hemisphere located $60 \mathrm{~cm}$ from the contralatcral cyc. Thc eye was focused at $60 \mathrm{~cm}$ by means of an appropriate contact lens. Visual stimuli could be presented up to $90^{\circ}$ out along the horizontal meridian, up to elevations of $55^{\circ}$ in the upper quadrant, and down to $60^{\circ}$ in the lower quadrant.

Histology. Histological procedures have been described in detail previously (Gattass and Gross, 1981). Electrolytic lesions were made at several sites along each penetration. Alternate $33 \mu \mathrm{m}$ frozen sections, either coronal or parasagittal, were stained for cell bodies with cresyl violet or for myelin with a modified Heidenhain-Woelcke stain (Gattass and Gross, 1981).

Unfolding of the cortical surface and visual maps. In order to obtain a "map" of the visual topography of V3 and V4 for each animal, we unfolded the prestriate cortex by building a 3-dimensional model and 

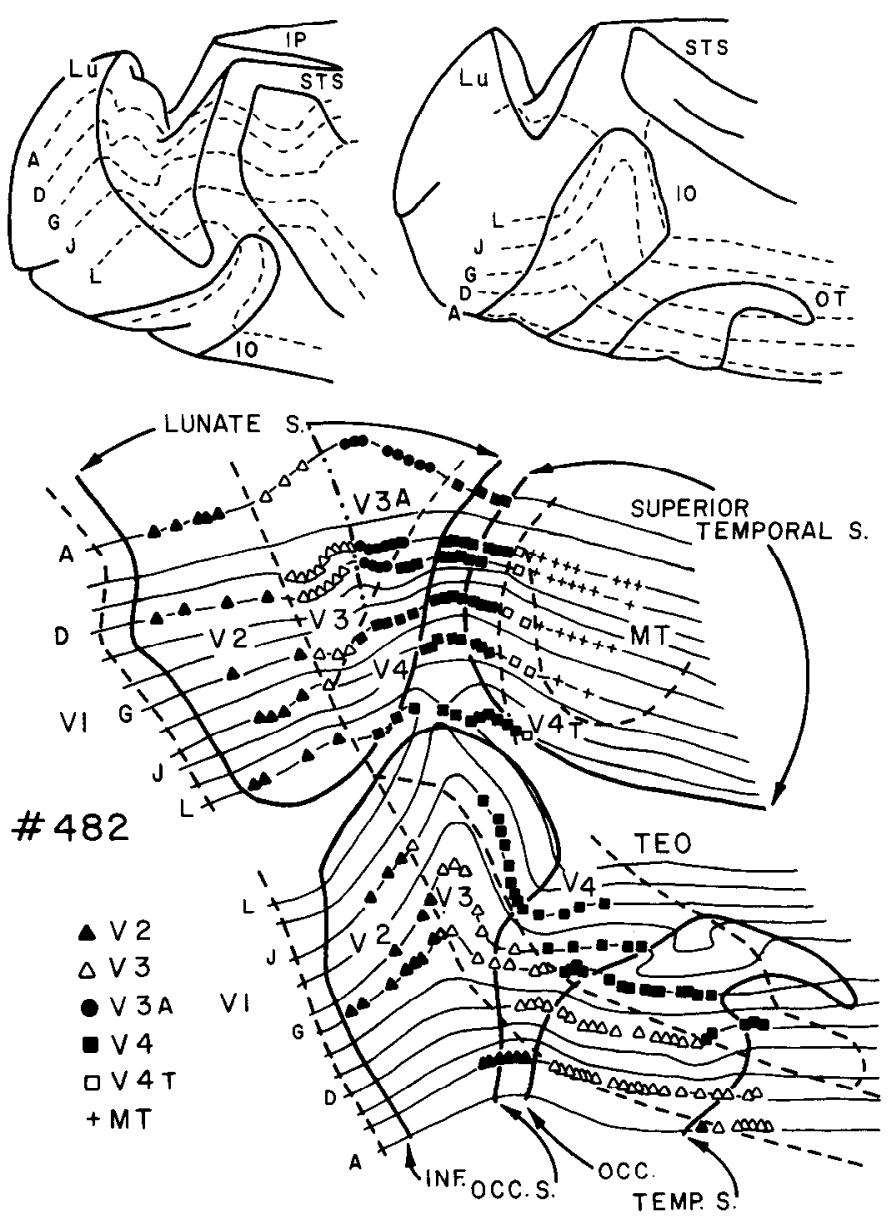

Figure 1. Flattened map of V3, V4, and vicinity showing recording sites and myeloarchitectonic borders between areas in animal 482 . The map was constructed, as described in the text, from tracings of sagittal sections $\mathrm{A}-\mathrm{L}$ at the levels indicated with dashed lines on the dorsolateral (top left) and ventrolateral (top right) views of the hemisphere, with sulci partially open. On the flattened map the myeloarchitectonically determined borders of areas V1, V2, V3, V3A, V4, V4T, MT, and TEO are indicated with dashed lines. The border between V3 and V3A was determined only on electrophysiological grounds. Symbols indicate the sites at which receptive fields were recorded. $I O$, inferior occipital sulcus; $I P$, intraparietal s.; $L u$, lunate s., $O T$, occipitotemporal s.; STS, superior temporal s.

then flattening it. First, myelin-stained sections through the occipital, parietal and temporal lobes, spaced $0.5 \mathrm{~mm}$ apart, were traced at $5 \times$ magnification. Then wires were bent to conform to layer IV of each section and connected with scaled cross pieces to form a 3-dimensional model of the posterior cortex. The model was then flattened, cutting the minimum number of cross pieces, to form a 2-dimensional representation. This method is equivalent to that of Van Essen and Zeki (1978) and Van Essen and Maunsell (1980). The myeloarchitectonic borders were marked on tracings of the flattened model, along with the recording sites projected onto layer IV, as shown in Figure 1. The representations of the visual field in the areas of interest were determined by marking the eccentricity of the receptive-field centers at each recording site on the flattened model and then fitting "isoeccentricity lines" by eye, as shown in Figure 2. We followed a similar procedure to determine the positions of the representations of the horizontal and vertical meridians. An example of a map with the meridians and isoeccentricity lines is shown in Figure 3.

\section{Results}

\section{Visual topography}

We will first summarize the location and visuotopic organization of V3 and V4, and then illustrate this organization with examples of the relationship between recording sites and thc location of the receptive fields recorded at those sites. In subsequent Results' sections, we consider receptive-field size and cortical magnification as a function of eccentricity, and describe the myeloarchitectonic characteristics of V3 and V4.

Area V3. Anterior to $\mathrm{V} 2$ there is a thin strip $(4-5 \mathrm{~mm})$ of cortex containing an organized representation of the contralateral visual field (Figs. 4, 5). Following Zeki (1969, 1978), who first proposed its existence in the macaque, we call this V3. The dorsal and ventral portions of V3 are discontinuous and, following the suggestion of Van Essen and his colleagues (Van Essen, 1985; Burkhalter et al., 1986) and the usage of Ungerleider and Desimone (1986b), we call the dorsal portion V3d and the ventral portion V3v. V3d lies in the fundus of the lunate sulcus and in the posterior portion of the annectant gyrus. In most, but not all, animals, it does not reach the medial surface, but terminates in the parietooccipital cleft (Fig. 6, left). V3v extends across both banks of the inferior occipital and occipitotemporal sulci (Fig. 6, right). Several different visual areas lie anterior to V3, as shown in Figures 4 and 6 . On the anterior border of V3d, moving lateral to dorsal, are areas V4, V3A, and PO (Zeki, 1971, 1978; Van Essen and Zeki, 1978; Covey et al., 1982; Gattass et al., 1985; Van Essen, 1985; Colby et al., 1988). Anterior to V3v lies the ventral portion of V4 (Gattass et al., 1985; Van Essen, 1985). As described in detail below, V3 can be distinguished myeloarchitectonically from all its neighbors except for parts of V3A.

We consider the visuotopic organization of V $3 \mathrm{v}$ first because it is somewhat more consistent across animals and simpler than that of $\mathrm{V} 3 \mathrm{~d}$. V $3 \mathrm{~V}$ contains a representation of about the central $35^{\circ}$ of the upper visual field (see Fig. 7). The representation of the horizontal meridian forms the posterior border of $\mathrm{V} 3 \mathrm{v}$ and is congruent with the representation of the horizontal meridian at the anterior border of the ventral portion of $\mathrm{V} 2$. The representation of the vertical meridian forms the anterior border and is congruent with the posterior border of the ventral portion of V4. This organization was found in the 6 animals studied; the interanimal variation in the location of $V 3 v$ is shown in Figure 6.

The visuotopic organization of $\mathrm{V} 3 \mathrm{~d}$ was both more variable and more complex than that of $\mathrm{V} 3 \mathrm{v}$. V3d contains a representation of about the central $40^{\circ}$ of the lower visual field (see Fig. 7). In all animals, the representation of the horizontal meridian forms the posterior border of V3d, and is congruent with that in $\mathrm{V} 2$, as is the case in $\mathrm{V} 3 \mathrm{~V}$. In 2 animals, the representation of the lower vertical meridian forms the anterior border of $\mathrm{V} 3 \mathrm{~d}$ (Fig. 8, left). However, in the 3 other animals, the situation at the anterior border of V3d is less straightforward. In these cases there is a partial split in the representation of the lower quadrant along a line that starts at the vertical meridian and is oblique to the horizontal meridian, and whose exact location varies from animal to animal. Thus, the anterior border of V3d consists of the representation of the vertical meridian divided into 2 portions, with a piece of the visual field intercalated between these portions (Figs. 8, right; 22, middle). A representation of the central $4^{\circ}-8^{\circ}$ of the vertical meridian abuts dorsal V4; the representation of the displaced portion of the visual field abuts $\mathrm{V} 3 \mathrm{~A}$; and the remainder of the representation of the vertical meridian abuts area $\mathrm{PO}$. The variability in the width of $\mathrm{V} 3 \mathrm{~d}$ observed from animal to animal appears to be a consequence of this arrangement (Figs. 6, 8). In fact, in one case V3d was so narrow at one point as to be almost divided into 2 portions. 


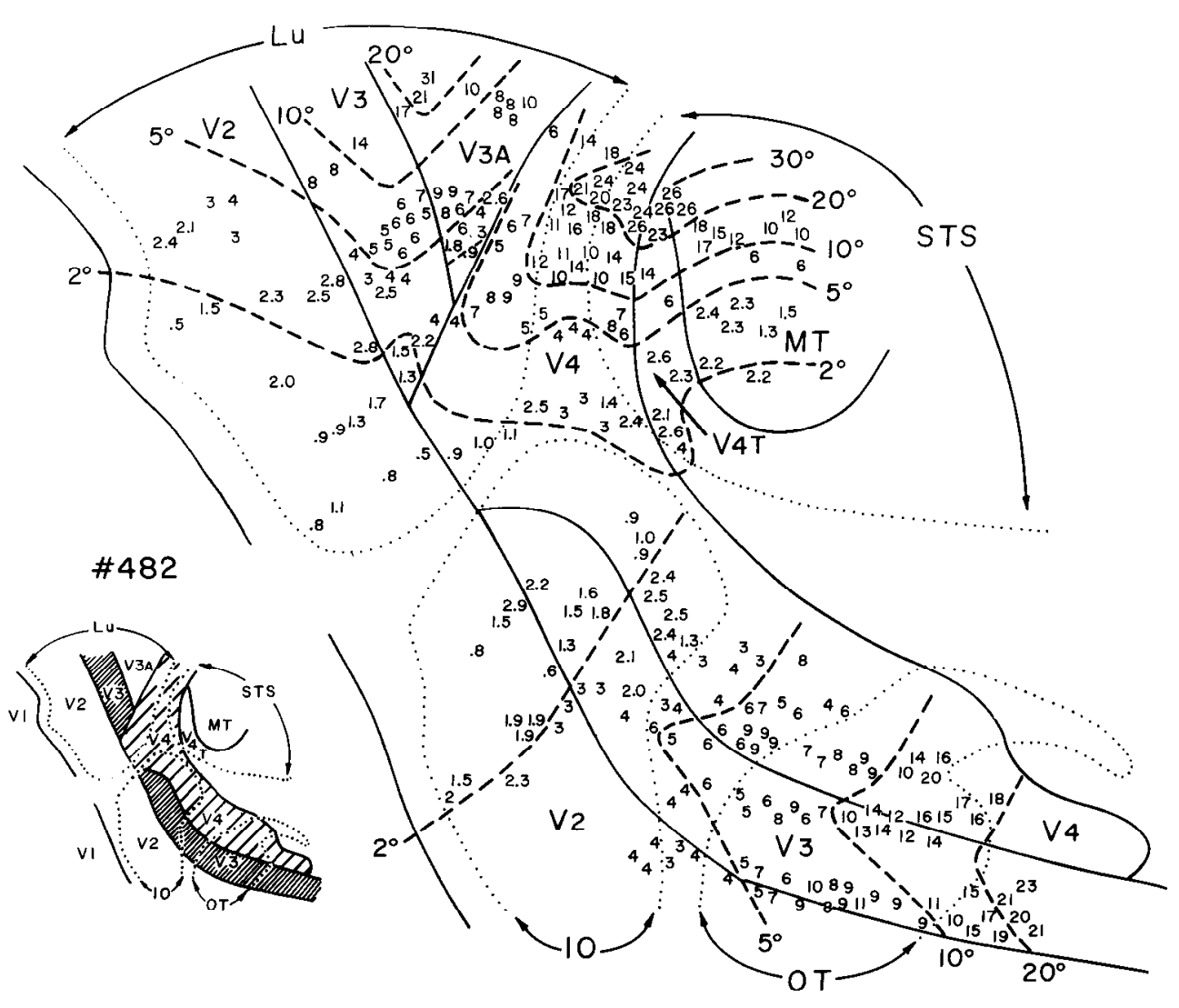

Figure 2. Flattened map of V3, V4 and vicinity showing how isoeccentricity lines were constructed. The map is the same as that shown in Figure 1 (bottom). At each recording site, the eccentricity of the receptive-field center recorded at that site is indicated in degrees. (Values of $3^{\circ}$ or higher are rounded to the nearest degree). Dashed lines represent isoeccentricity lines fitted by eye to these values. Dotted lines show the open sulci, and solid lines the area boundaries, as in Figure 1. Inset shows the same flattened map with V3 and V4 shaded differentially to show area boundaries more clearly.
On the lateral surface, anterior to the foveal representation in V1, where V2, V3d, and V4 meet, the receptive fields are located within a few degrees of the center of gaze, and it is not possible to differentiate these areas on the basis of receptivefield topography or receptive-field size. In this region, the borders between the areas could be drawn only on a myeloarchitectonic basis. By these criteria, V4 abuts V2, thereby separating V3d from V3v, as shown in Figure 2, lower left.

As discussed below, receptive-field size in V3 increases markedly with increasing eccentricity. However, even at the more peripheral eccentricities, the nasal borders of the receptive fields do not extend across the vertical meridian into the ipsilateral field by more than about $3^{\circ}-4^{\circ}$. A consequence of this is that, with increasing eccentricity, the vertical meridian is not represented by fields centered at the meridian; instead, it is represented by the nasal portion of the receptive fields whose centers are located away from the meridian (see Fig. 14). This is also true in peripheral V2 (Gattass et al., 1981), MT (Gattass and Gross, 1981), and V4.

Area V4. Anterior to V3 there is a strip of myeloarchitectonically distinct cortex 5-8 $\mathrm{mm}$ wide (Figs. 4,5 ). It contains an organized representation of the contralateral visual field. We call it V4 because it has about the same location, at least dorsally, as the area called V4 or the V4 complex by Zeki $(1971,1977)$. Dorsally, V4 extends from the anterior bank of the lunate sulcus across the prelunate gyrus onto the lip of the posterior bank of the superior temporal sulcus (Fig. 6, left). Ventrally, it extends across the inferior occipital and occipitotemporal sulci (Fig. 6, right).

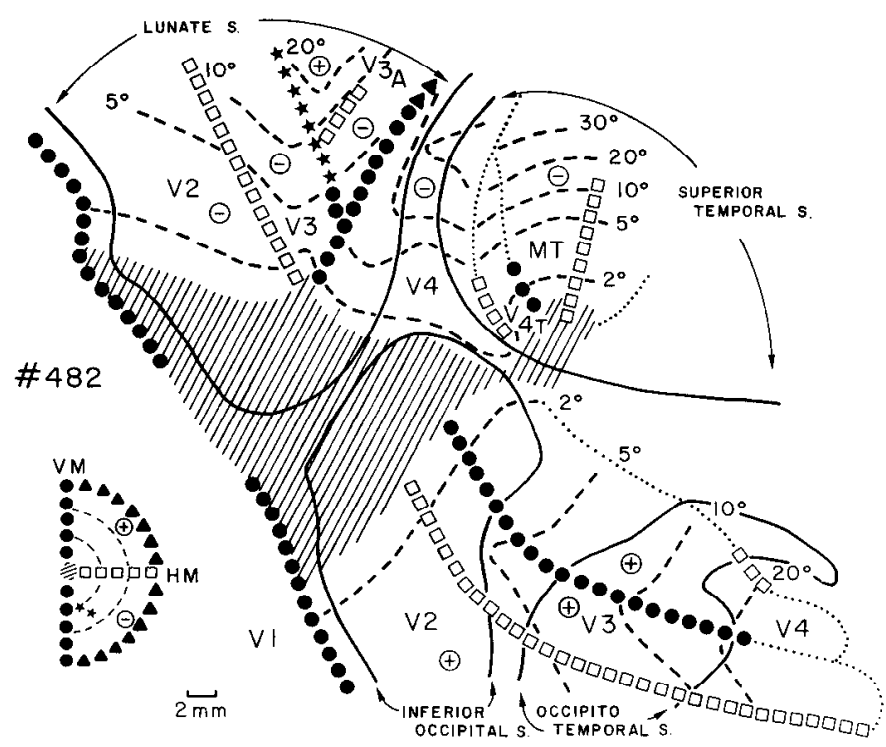

Figure 3. Visual topography of V3, V4, and neighboring areas of animal 482 , showing the representation of the upper field $(+)$, lower field $(-)$, vertical meridian (black circles), horizontal meridian (squares), center of gaze (hatched), and isoeccentricity (dashed) lines. Dotted lines represent myeloarchitectonic borders. The anterior border of V3d (stars) corresponds to the region of the visual field shown with stars in the representation of the contralateral hemifield in the inset in the lower left. 

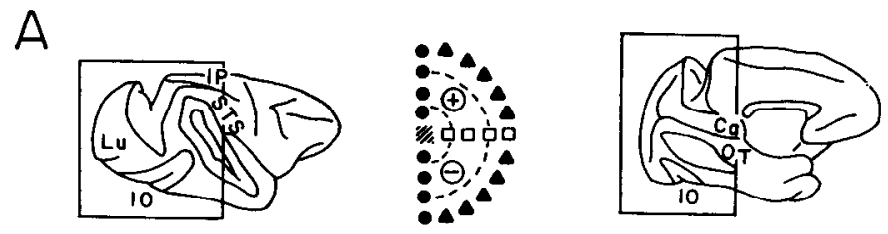

Figure 4. Summary of location and visual topography of V3 and V4 based on data from 12 animals. $A$, Lateral and medial views with sulci partially opened up. The regions in the rectangles are shown enlarged in $B$. $B$, Lateral and medial views showing representations of the vertical meridian (black circles), the horizontal meridian (squares), the center of gaze (hatched), isoeccentricity lines (dashed), and the myeloarchitectonic borders (dotted lines) for V2, V3, V3A, V4T, and MT. $\mathrm{Ca}$, calcarine sulcus. See also legends for Figures 1 and 3.

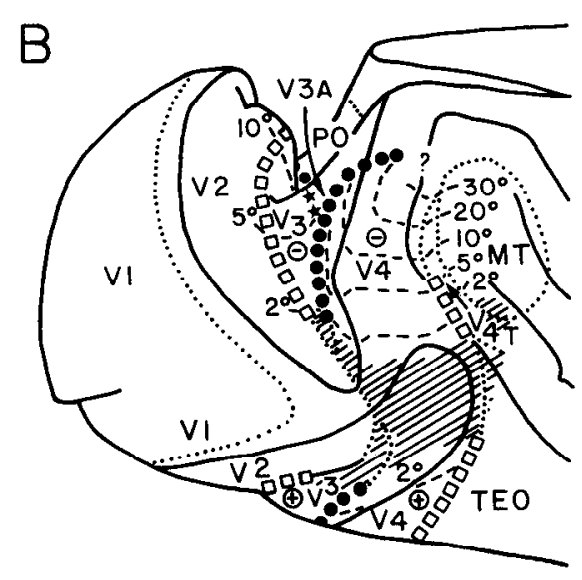

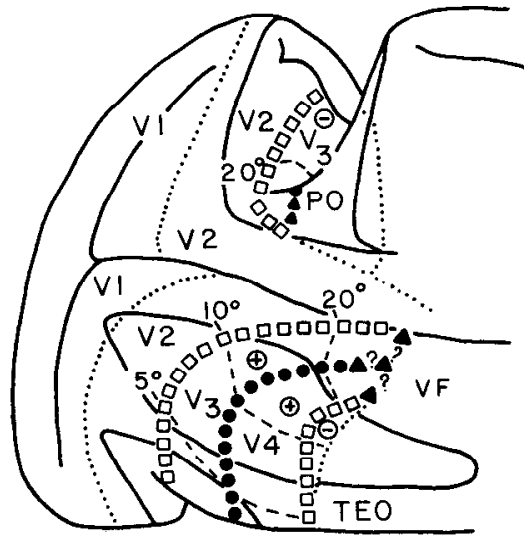

V 4 contains a representation of about $35^{\circ}-40^{\circ}$ of the visual field, as shown in Figure 7. The representation of the upper visual field is located ventrally. Most of the representation of the lower visual field is located dorsally, but a small and variable portion is located close to the anterior border of ventral V4. This portion of the lower field located ventrally includes the region adjacent to the horizontal meridian beyond an eccentricity of about $5^{\circ}$, as shown in Figures 9, 14, 15, and 18. Thus, in V4 the split of the representation of the contralateral visual field does not take place entirely along the horizontal meridian, as it does in V2 and V3; rather, beyond an eccentricity of about

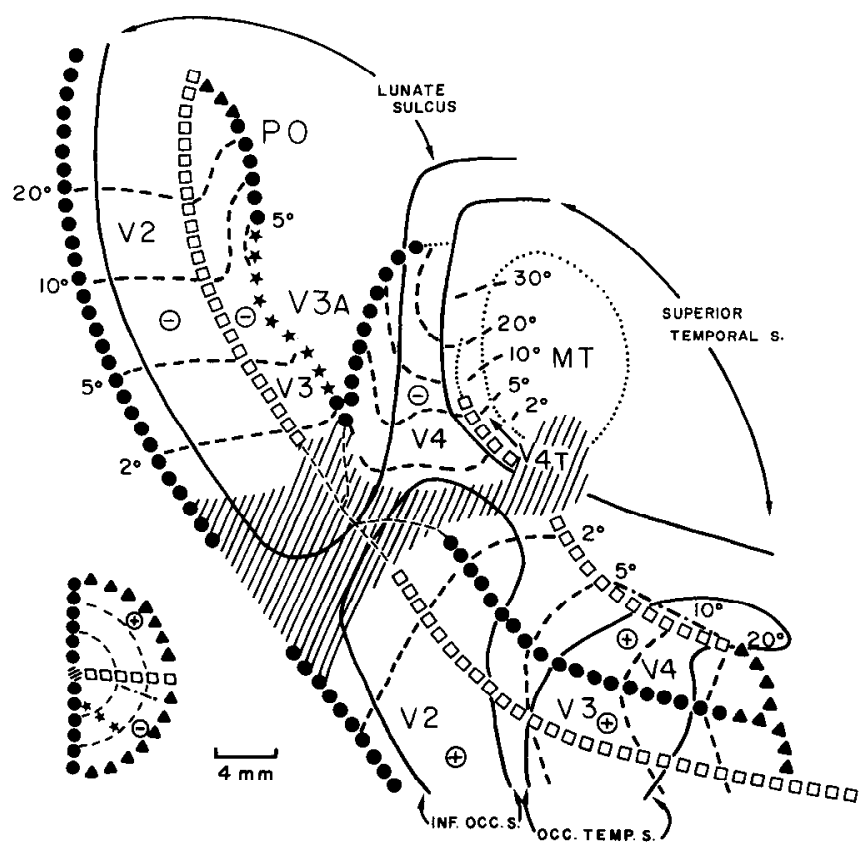

Figure 5. Flattened map showing the visual topography of V3 and V4, based on data from 12 animals. See also legend to Figure 3. $5^{\circ}$ it takes place along a line oblique to the horizontal meridian, as diagrammed in the lower part of Figure 22.

The posterior border of $\mathrm{V} 4$ corresponds to the representation of the vertical meridian (Figs. 4, 5). Ventrally, the representation of the vertical meridian in V4 is adjacent to and congruent with that in V3, up to an eccentricity of about $20^{\circ}$. Dorsally, the posterior border of $\mathrm{V} 4$ corresponds to the representation of the central portion of the vertical meridian $\left(2^{\circ}-8^{\circ}\right)$ abutting V 3 and, more medially, V3A. As was the case for V3, with increasing eccentricity the centers of the receptive fields are not located on the vertical meridian, although their borders are located near or on this meridian (cf. Fig. 10).

In $\mathrm{V} 4$, the visuotopic organization of the dorsal portion was more complex and more variable among animals than that of the ventral surface. Overall, the visuotopic organization of V4 was "cruder" and "noisier" than that in V3, and more so than in either V2 or V1. For example, there were reversals in the systematic progression of receptive fields, and receptive fields in the same location in the visual field could be found at recording sites $1 \mathrm{~mm}$ or further apart (see Figs. 16, 18).

Several areas border V4 anteriorly (Figs. 4, 5). The anterior border of dorsal V4 abuts area V4T. Area V4T (T for transitional) is an approximately $2-\mathrm{mm}$-wide strip that lies along part of the posterior border of area MT. It is myeloarchitectonically distinct from both V4 and MT and contains a representation of the lower visual field. Area V4T appears to correspond to part of the "colour coding area of the superior temporal sulcus" described by Zeki (1977). This transitional area between V4 and MT was subsequently noted by Schein et al. (1982), Maguire and Baizer (1984), and Ungerleider and Desimone (1986b; Desimone and Ungerleider, 1986). The latter showed that this area receives a projection from MT (which V4 does not). At the border of V4 and V4T, there is a representation of the horizontal meridian with receptive-field centers located at $4^{\circ}-8^{\circ}$, and temporal borders at up to $18^{\circ}$ along the horizontal meridian.

It was usually difficult to delimit precisely the dorsal border of V4 by myeloarchitectonic methods alone. In Figure 10 the approximate region of the dorsal border of V4 is marked with 

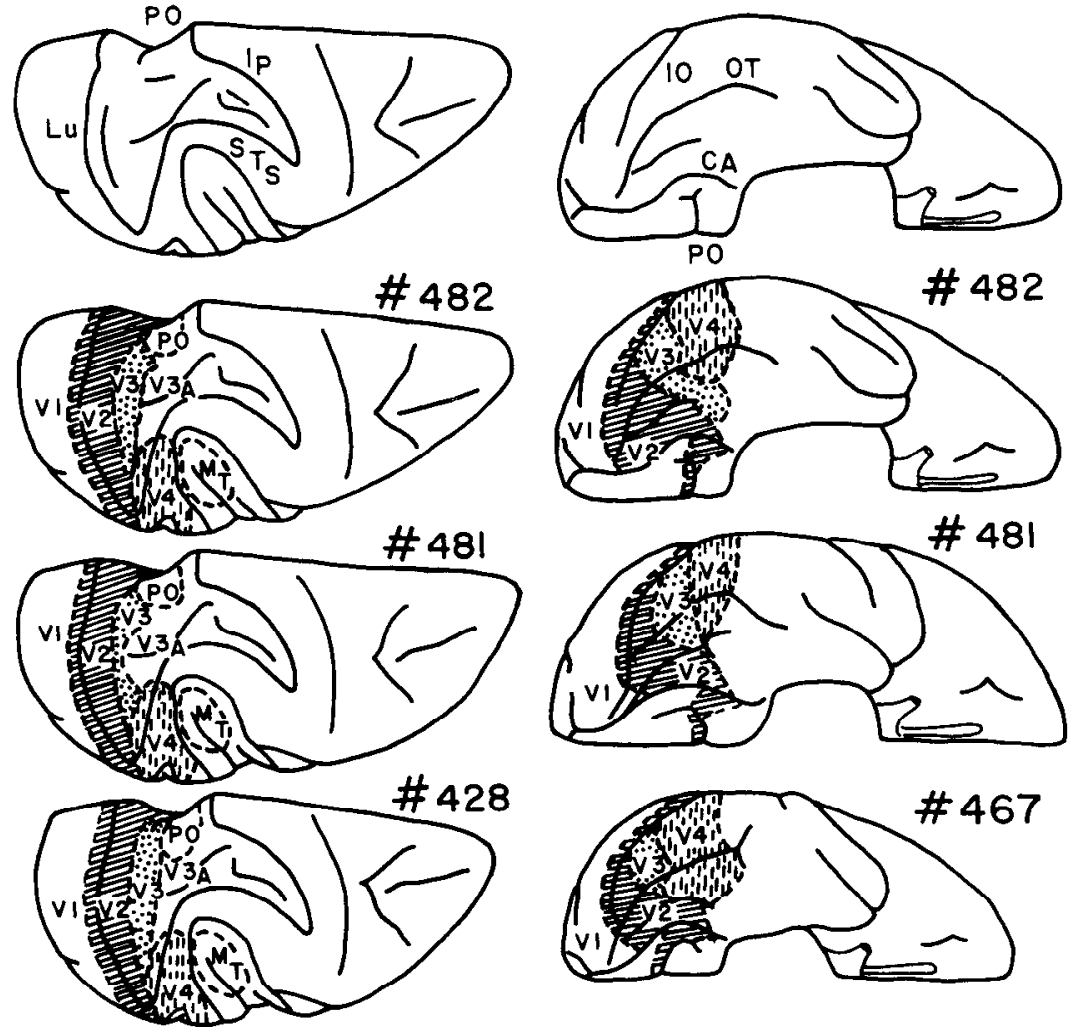

Figure 6. Variation among 4 animals $(482,481,428$ and 467$)$ in the location of V3 and V4 shown in dorsal views in which sulci have been opened (left) and in ventral views (right). For abbreviations, see Figure 1. a dashed line and a question mark. Note that in section B in moving from site 6 (which is clearly in myeloarchitectonic V4) to sites 7 and 8 in the border region, there is both a discontinuity in the location of the receptive-field centers and a marked increase in receptive-field size. Thus, the dorsal border of V4 at this point appears to be between site 6 and sites 7 and 8 .

Ventrolaterally, V4 borders area TEO. This area extends from the ventral bank of the superior temporal sulcus to the lateral bank of the occipitotemporal sulcus. It is distinguishable from its neighbors on the basis of cytoarchitecture, myeloarchitecture, connections, visuotopic organization, and the behavioral effects of lesions (Iwai and Mishkin, 1969; Mishkin, 1972; Fenstemaker et al., 1984-1986). There is a representation of the horizontal meridian at or near the border of V4 and TEO. However, as mentioned above, in all 4 cases there was a representation of part of the inferior visual field in ventral V4. This representation, along with a varying amount of representation of the horizontal meridian, forms the anterior border of ventral V4. Owing to the partial representation of the inferior visual field in ventral $\mathrm{V} 4$, the entire horizontal meridian is not represented in both dorsal and ventral V4, as it is in dorsal and ventral V2 and V3. Rather, the entire horizontal meridian is represented in ventral V4, except for the central portion, which is also represented in dorsal V4.

Medial to the occipitotemporal sulcus, V4 borders on visual cortex that we previously named area VF (Gattass et al., 1985). VF includes a representation of at least part of the lower visual field. In addition, at the anterior border of $\mathrm{V} 4$, in the region of the occipitotemporal sulcus, there appears to be at least one representation of the upper quadrant that is separable from both TEO and VF (R. Gattass and A. P. B. Sousa, unpublished observations). One or more of these areas may correspond to area PT, described by Felleman et al. (1986).

\section{Receptive-field sequences in V3 and V4}

In this section we illustrate the visuotopic organization of V3 and V4 by showing the relationship between receptive-field location and recording sites in representative parasagittal sections.

In Figure 11, the recording sites are in V2, V3d, V4, V4T, and MT. Starting in V2 near the border with V1 (site 1) and moving down the posterior bank of the lunate sulcus, the fields move from the vertical meridian to the horizontal meridian (sites 1-4). Crossing the myeloarchitectonic border into V3d (site 5) and moving toward the anterior border of V3d (sites 6$10)$, the progression of receptive fields reverses and the field centers move from the horizontal meridian towards the vertical
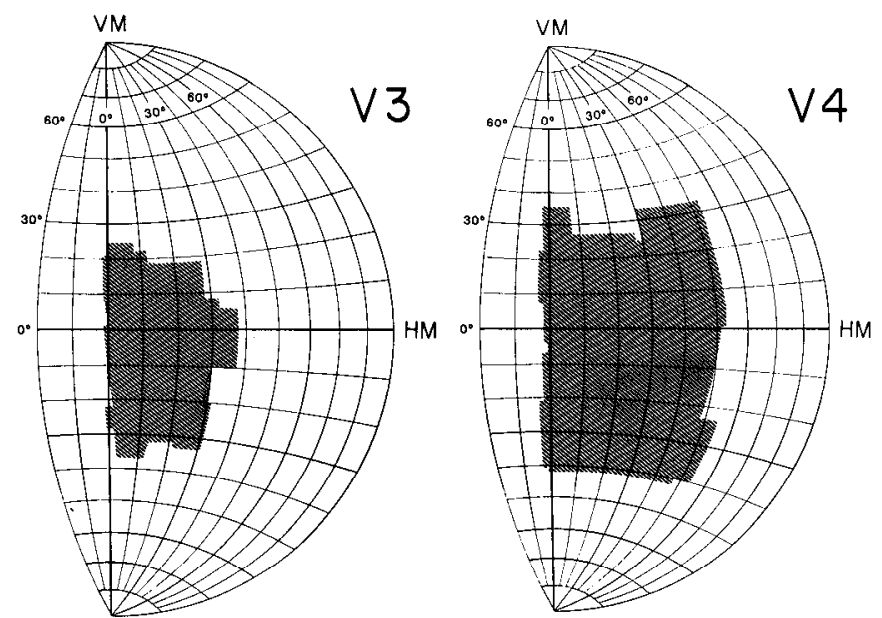

Figure 7. Extent of the visual-field representation in V3 and V4. The shaded areas were obtained by delineating the external borders of superimposed receptive fields recorded from 11 animals. 

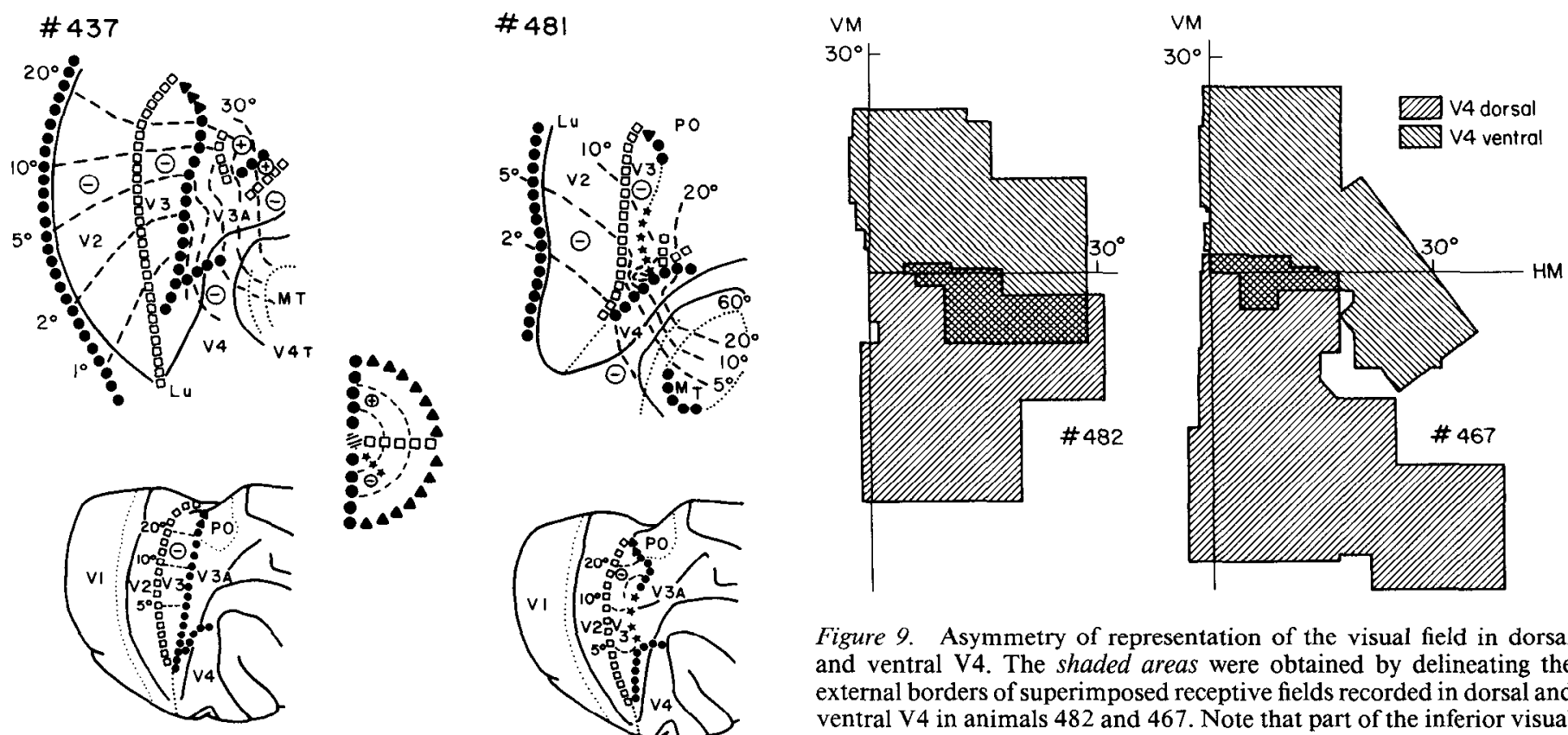

Figure 9. Asymmetry of representation of the visual field in dorsal and ventral V4. The shaded areas were obtained by delineating the external borders of superimposed receptive fields recorded in dorsal and ventral V4 in animals 482 and 467 . Note that part of the inferior visual field (beyond approximately $5^{\circ}$ ) is represented in ventral V4.

Figure 8. Visual topography of V3d. Top, Flattened maps showing the visual topography of V3d in a case (animal 437) where the anterior border of $V 3 \mathrm{~d}$ consists only of the representation of the vertical meridian (left), and in one (481) in which the anterior border is more complex (right). Bottom, Dorsal views showing the 2 variants of V3d. For symbols see Figure 3.

meridian. Crossing the myeloarchitectonic border into V4 (sites 11 and 12) and moving dorsally onto the prelunate gyrus, the receptive fields move from the center of gaze out into the periphery (sites 11-22). Crossing the myeloarchitectonic border into V4T (site 23), the field progression reverses again and the centers move in from the periphery (sites 23-24). Moving down the lower bank of STS and crossing the myeloarchitectonic border of MT, the fields move from the periphery of the inferior visual field (site 25) across the horizontal meridian into the superior visual field (sites 25-29), as has been previously dcscribed (Gattass and Gross, 1981; Van Essen et al., 1981).

More ventrally, at the V4/V4T border the centers of the receptive fields are found at the horizontal meridian, and at the V4T/MT border they are found at the vertical meridian. However, at the level of this section the receptive fields in V4, V4T, and MT are so large that the receptive-field centers at these borders are displaced from the meridians. Moreover, at all levels the representations of the visual field remain congruent across both the V4/V4T and the V4T/MT borders.

Figure 12 presents data from a more medial section from the same animal. The section now passes through V3A as well as V2 and V3d. Starting again in V2, near its border with V1 (site 1), and moving down the posterior bank of the lunate sulcus, the fields move from the vertical meridian toward the horizontal meridian (sites 1-9). Crossing the myeloarchitectonic border into $\mathrm{V} 3 \mathrm{~d}$, we begin near the representation of the horizontal meridian at site 10 (asterisk). Moving anteriorly within V3d from site 11 to site 14 , the receptive fields move from the horizontal to the vertical meridian at the anterior border of V3d. Moving dorsally within V3d from site 10 to sites $15-21$, the receptive fields move toward the periphery. At site 22 , the field progression reverses and the fields move back toward the center

of gaze (sites 22-27). Starting with site 22 or nearby, we are presumably in V3A. We could discern no consistent myeloarchitectonic border in this vicinity; thus, the sole evidence that we are in a new area is the reversal of field progression and the re-representation of the visual field.

Figure 13 is a section from a different animal, located more medially than the section illustrated in Figure 12. It again includes parts of V2, V3d, and V4, but now it passes through the regions of representation of both the inferior and superior visual fields in V3A. As in the previous sections, we start in V2 at the vertical meridian (site 1) and move toward the horizontal meridian ( (and is even narrower at more medial levels). Within V3d there are only 2 recording sites ( 9 and 10$)$, and the tendency, moving anteriorly, is for the receptive-field centers to move away from the horizontal meridian. Starting at sitc 11 , the receptive-ficld progression reverses and, as we move dorsally up the anterior bank of the lunate sulcus, the receptive fields move across the horizontal meridian into the superior visual field. We are now in V3A and presumably its border with V3d is in the vicinity of sites 10 and 11 . Sites 18-22 are in more anterior regions of $\mathrm{V} 3 \mathrm{~A}$ in the lateral bank of the intraparietal sulcus, where the periphery of the superior visual field is represented (R. Gattass, unpublished observations). The myeloarchitectonic border of $\mathrm{V} 3 \mathrm{~A}$ and V4 is between sites 17 and 23. Starting at site 23 in $\mathrm{V} 4$ and moving dorsally onto the prelunate gyrus, the receptive fields move into the periphery.

Figures 14 and 15 illustrate the borders of V3v and V4 on the ventral surface. In Figure 14, site 1 is near the V2/V3v border and its receptive field is on the horizontal meridian. Moving anteriorly through the occipitotemporal sulcus, the receptive fields move toward the vertical meridian (sites 1-9). Crossing the myeloarchitectonic border with V4 between sites 9 and 10 , the field progression reverses and the fields move from the vertical meridian (site 10) to below the horizontal meridian (site 14). Note that the centers of the receptive fields located at the border between $\mathrm{V} 3 \mathrm{~V}$ and $\mathrm{V} 4$, which is the representation of the vertical meridian (sites 9 and 10), are not actually on the vertical 

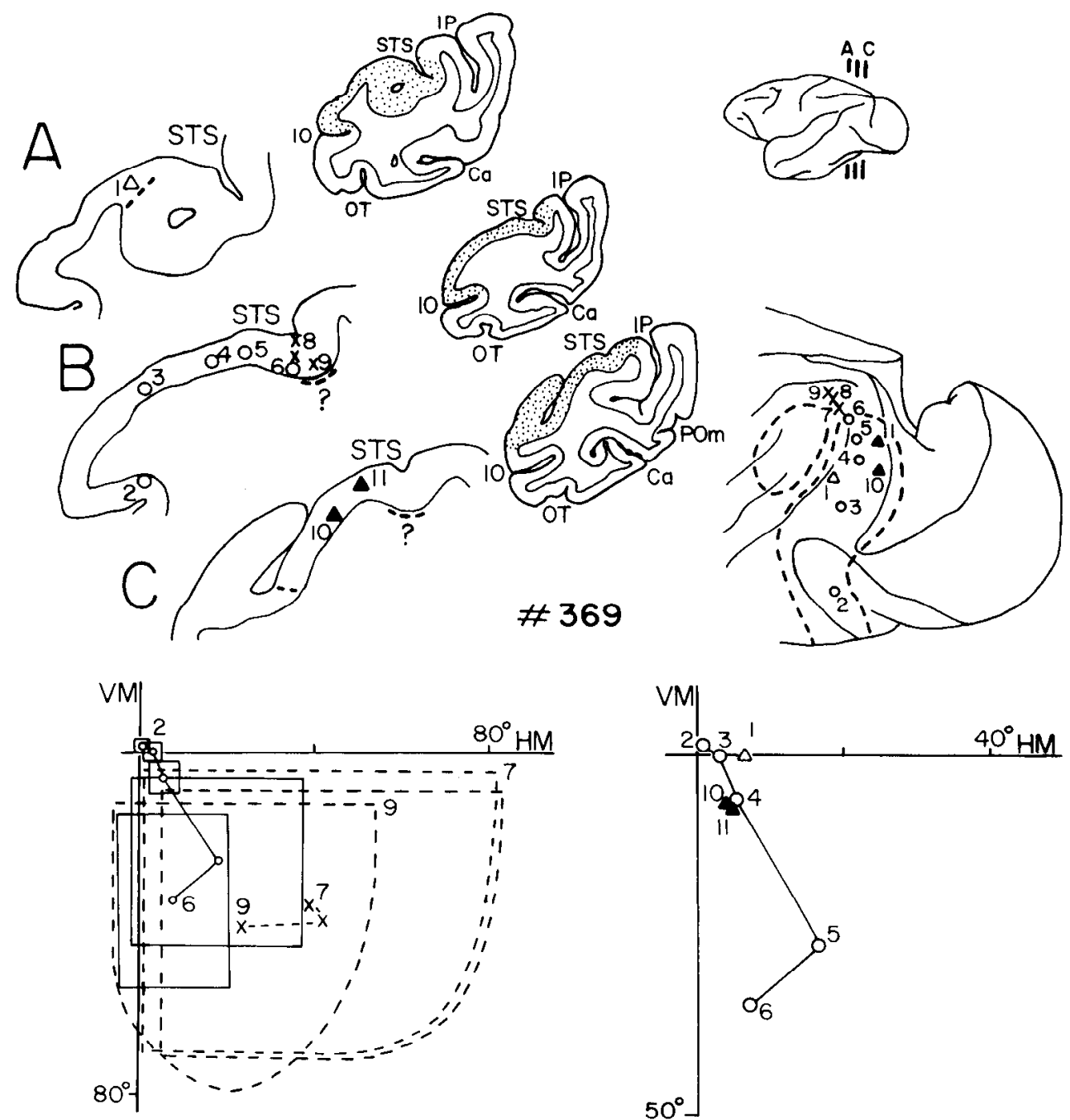

Figure 10. Location of receptive fields in dorsal $\mathrm{V} 4$ and adjacent cortex in animal 369 recorded at sites indicated on the enlarged portions (shaded) of the coronal sections cut at the levels indicated on the lateral view of the brain shown in the upper right. Sites 1-6, 10 and 11 are within V4, and sites 7-9 are dorsal to V4. The borders of V4 in the sections are shown with dashed lines the question marks indicate that the dorsal border could not be defined precisely on myeloarchitectonic grounds. Lower right, Receptive-field centers recorded in V4 at sites indicated in sections A, B, and C. Lower lefl, Receptive fields and receptive-field centers recorded at sites indicated in section B. The fields and centers recorded within V4 (at sites 2-6) are shown with solid lines and circles, respectively, and those recorded outside of V4 (at sites 7-9) are shown with crosses and dashed lines. Abbreviations as in Figure 1 meridian, although the receptive fields do include the vertical meridian. As described above, this is usually the situation at eccentricities beyond about $5^{\circ}$. Furthermore, note that at site 14 , near the anterior border of $\mathrm{V} 4$, as determined by myeloarchitectonic criteria, the entire receptive field is in the inferior visual field. As mentioned above, some representation of the peripheral inferior visual field at the anterior border of ventral $\mathrm{V} 4$ was characteristic of all the cases examined.

Figure 15 illustrates results from a more lateral section from the same animal. At site 1 , located in V3v near its border with $\mathrm{V} 2$, the receptive field is near the horizontal meridian. Moving anteriorly, along the anterior bank of the inferior occipital sulcus, the fields shift toward the vertical meridian (sites 1-7) but remain near the center of gaze. Moving across the myeloarchitectonic border into $\mathrm{V} 4$, the field progression reverses and the fields move from the vertical meridian (site 8 ) to below the horizontal meridian (site 16) at the anterior border of V4. Moving across the border with TEO, there is another reversal of field progression (sites 17-19).

Figure 16 shows the overall visual topography of the dorsal portion of V4 in a series of parasagittal sections from animal 482. In each section, moving anteriorly up the anterior bank of the lunate sulcus from the border of V3 and V3A with V4 onto the prelunate gyrus toward the anterior border of V4, the receptive fields shift from the vertical meridian toward the hor-
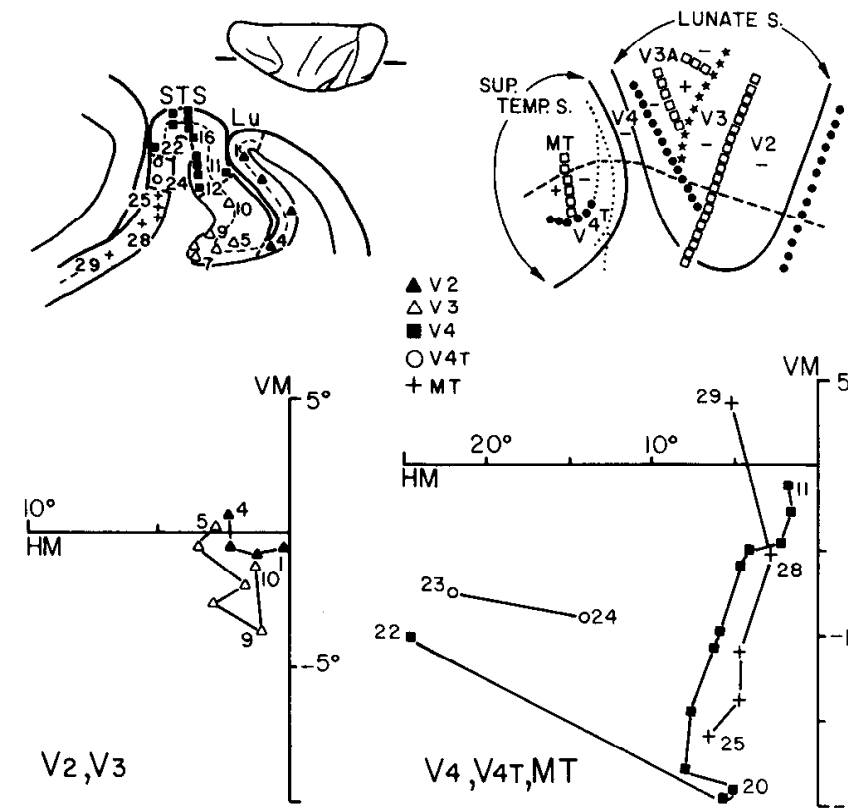

$$
\begin{aligned}
& \Delta \vee 2 \\
& \Delta \vee 3 \\
& \text { QV4 } \\
& \text { OV4T } \\
& +M T
\end{aligned}
$$$$
+M T
$$

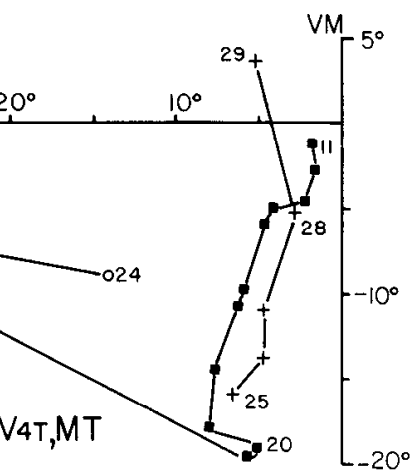

Figure 11. Location of receptive-field centers in V3d and V4 and adjacent areas recorded at sites indicated in the parasagittal section (animal 482). The drawing in the upper right is a schematic representation of the visual topography of V3, V4, and adjacent areas showing the level of the section (dashed line). See also legends to Figures 1 and 3. 

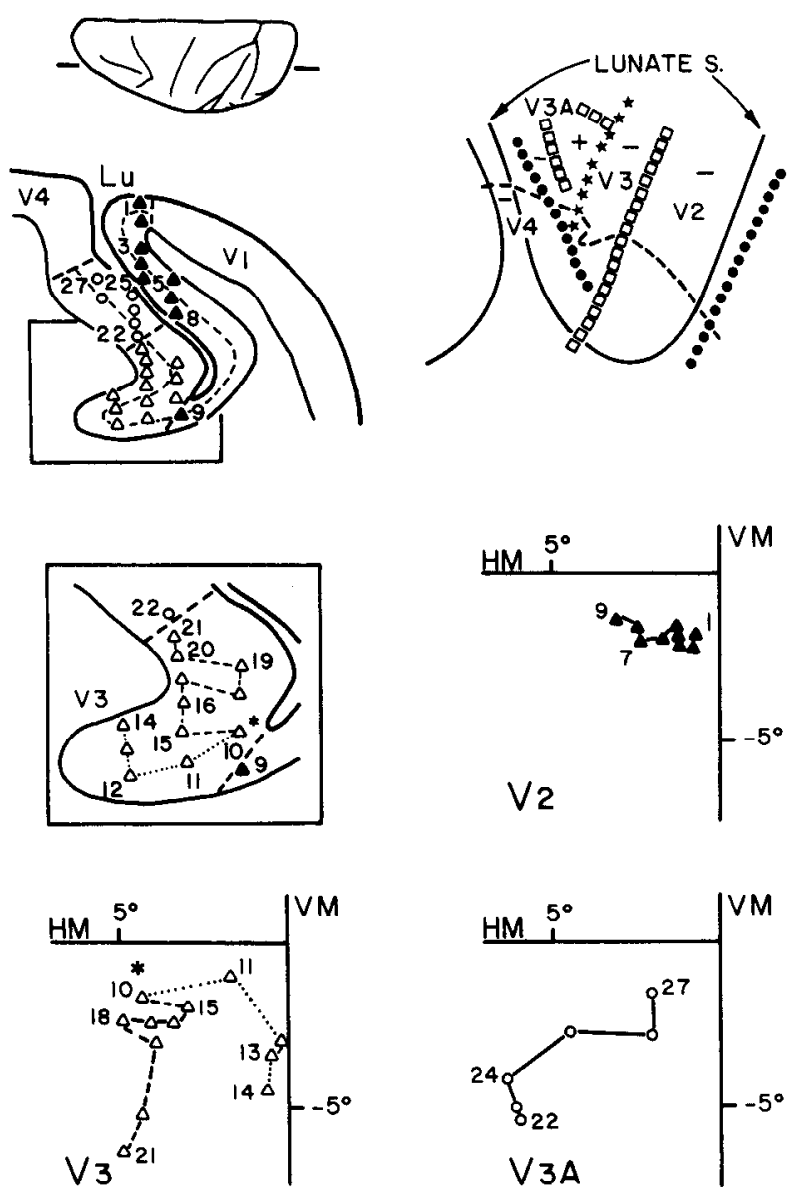

Figure 12. Location of receptive-field centers in V3d and adjacent areas, recorded at sites indicated in the parasagittal section from the same animal (482) illustrated in Figure 11. See also legend to Figures 1 and 3 .

izontal meridian. Furthermore, moving from lateral sections to the medial sections (A to $F$ ), the receptive fields shift from near the center of gaze into the periphery. As in ventral V4, the centers of the receptive fields recorded at the posterior border of V4 (which corresponds to the representation of the vertical meridian) do not lie on the vertical meridian, but the borders of the fields do include this meridian. The area covered by the receptive fields recorded in dorsal V4 for this animal is shown in Figure 9, left. An example of the crudeness of the visuotopic organization of $\mathrm{V} 4$ is that sites as much as $1.5 \mathrm{~mm}$ apart may have receptive fields at similar locations in the visual field (e.g., sites $13-16$ in section $C$ and sites $26-29$ in section D).

Figure 17 illustrates the visual topography of dorsal V4 in a series of parasagittal sections from another animal (467). Again, the centers of the receptive fields recorded at the posterior border of V4 lie near, but not on, the vertical meridian. In this animal the representation of a significant part of the inferior quadrant is located in ventral V4 rather than in dorsal V4 (cf. Fig. 9, right, with Fig. 17).

Figure 18 shows the overall visual topography of the ventral portion of $\mathrm{V} 4$ in a series of parasagittal sections from the same animal illustrated in Figure 17. Moving anteriorly from the border of $\mathrm{V} 3$ with $\mathrm{V} 4$ to the anterior border of $\mathrm{V} 4$, the receptivefield centers move from the vertical meridian toward the horizontal meridian. In fact, near the anterior border of V4, as noted previously, the receptive-field centers actually lie in the

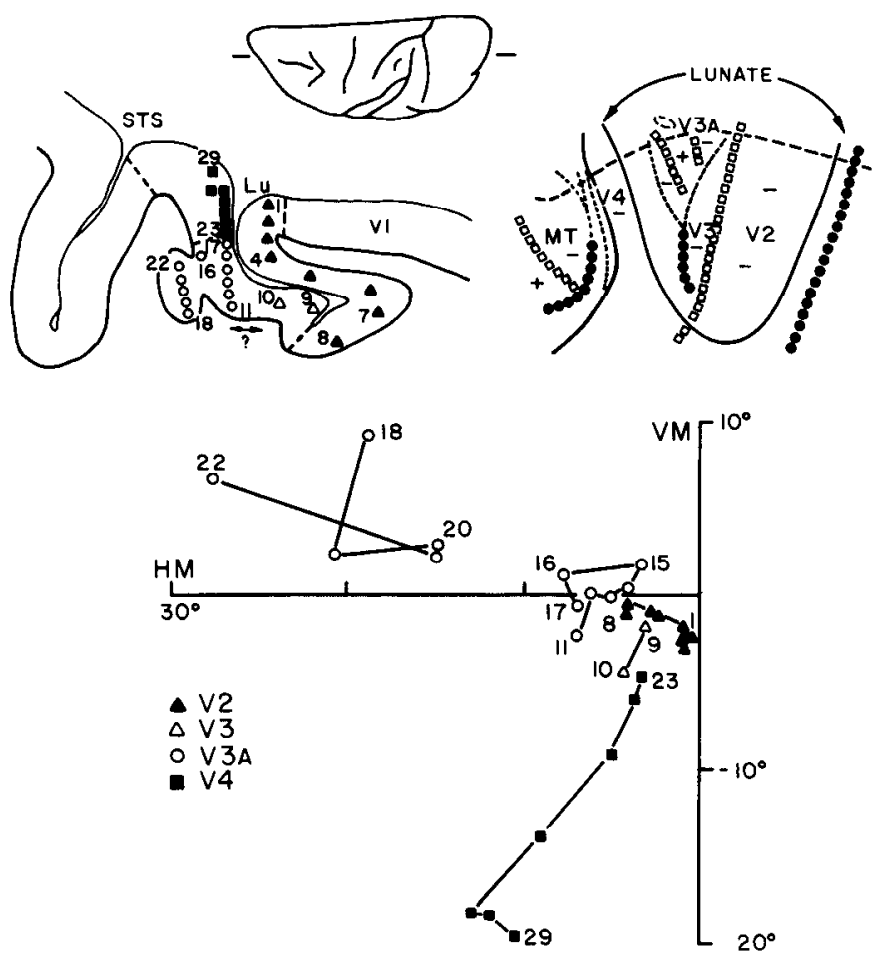

Figure 13. Location of receptive-field centers in V3d and V4 and adjacent areas recorded at the sites indicated in the parasagittal section from animal 481. See also legend to Figures 1 and 3.

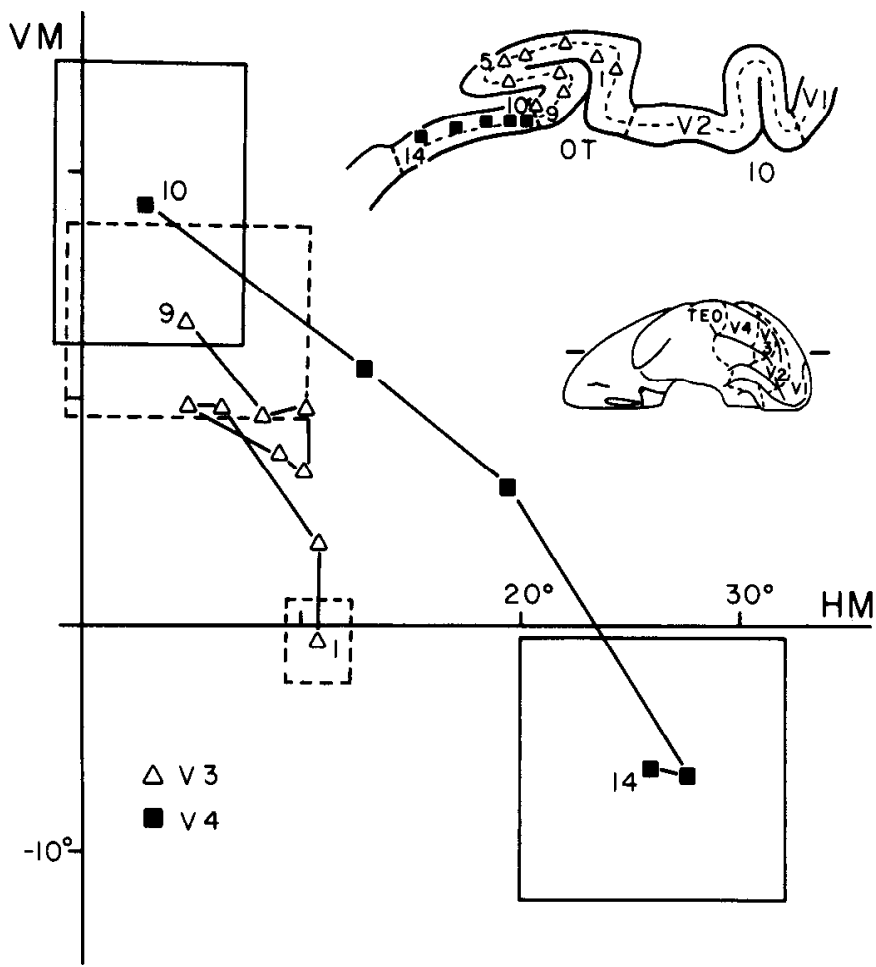

Figure 14. Location of receptive-field centers in V3v and V4 recorded at the sites indicated in the parasagittal section, cut at the level indicated in the ventral view of the brain (animal 467). The entire receptive fields, as well as the receptive-field centers, are shown for sites $1,9,10$, and 14. 


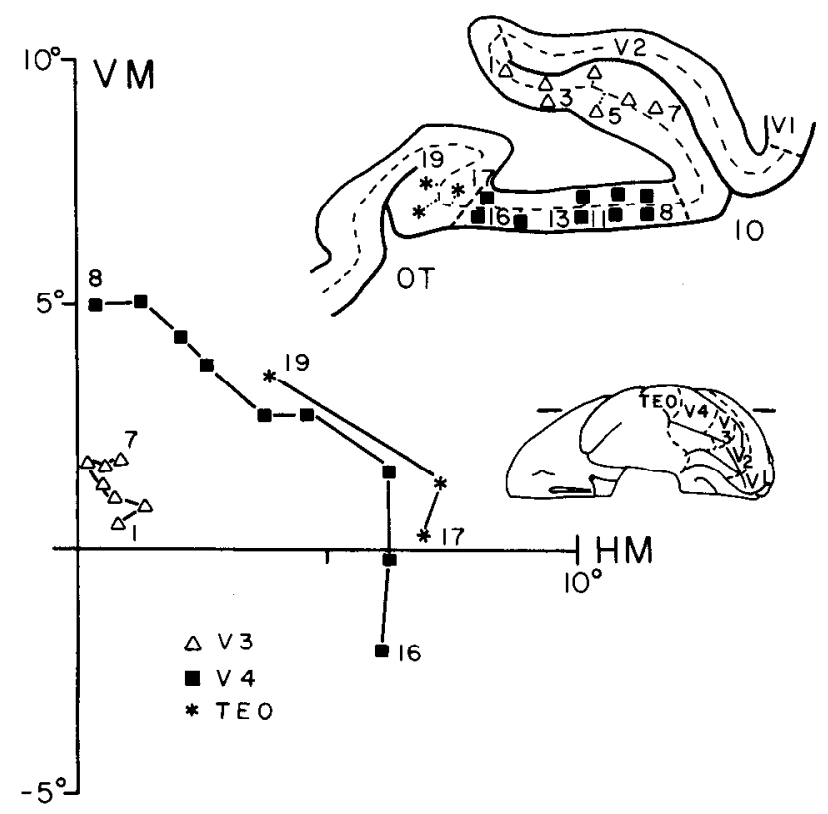

Figure 15. Location of receptive-field centers in V3v and V4 and area TEO recorded at the sites indicated in the parasagittal section from the same animal (467) shown in Figure 14. inferior visual field. Moving from the lateral to the medial sections (D to A), the receptive-field centers move from the center of the visual field to the periphery. Note that the progressions are more orderly and there are less re-representations than in dorsal V4 (Fig. 16). This may reflect both a more orderly visuotopic organization in ventral than in dorsal $\mathrm{V} 4$ and the fact that the penetrations in ventral V4 are more orthogonal to the cortical surface.

\section{Changes in neuronal properties with eccentricity}

Receptive-field size. Receptive-field size, i.e., the square root of receptive-field area, is plotted as a function of the eccentricity of the receptive field-center for V3d and V3v in Figure 19A, and for dorsal and ventral V4 in Figure 19B. These measurements of multiunit receptive-field size were carried out with one electrode with a constant impedance $(0.7 \mathrm{M} \Omega)$, since the size of multiunit receptive fields varies with electrode impedance. A straight line was fitted to each set of data using the method of least squares. We found that in both areas, receptive-field sizes increase markedly with increasing eccentricity.

There was no significant difference between the $y$-intercepts $(t=0.16, p>0.2)$ or the slopes $(t=1.15, p>0.2)$ of the regression lines for V3d and V3v. Similarly, there was no significant difference between the $y$-intercepts $(t=1.46, p>0.1)$
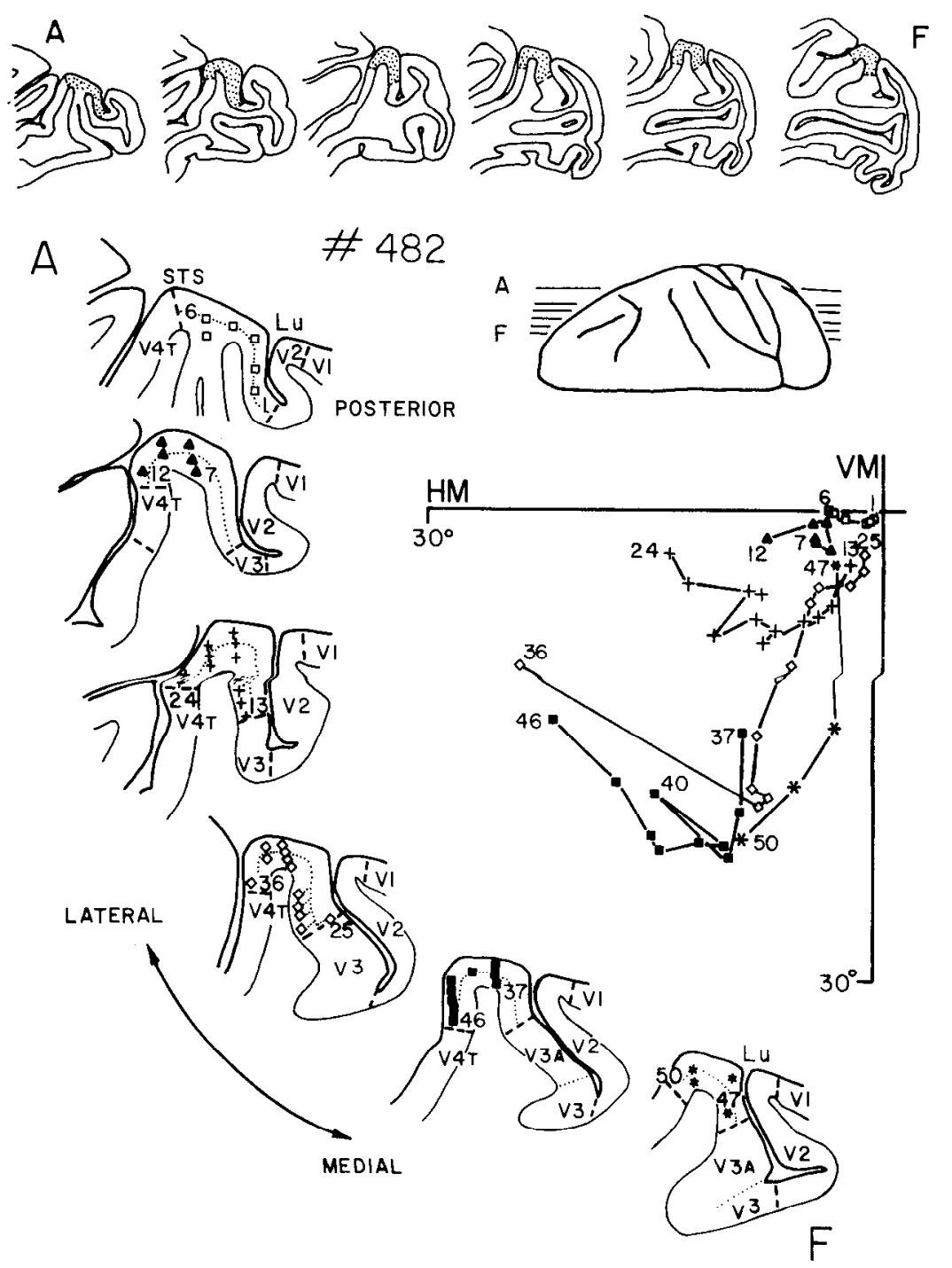

Figure 16. Location of receptive-field centers in dorsal V4 (shaded) recorded at the sites indicated in a series of parasagittal sections $(\mathrm{A}-\mathrm{F})$, cut at the levels indicated in the dorsal view of the brain (animal 482). For abbreviations see Figures 1 and 5 . 

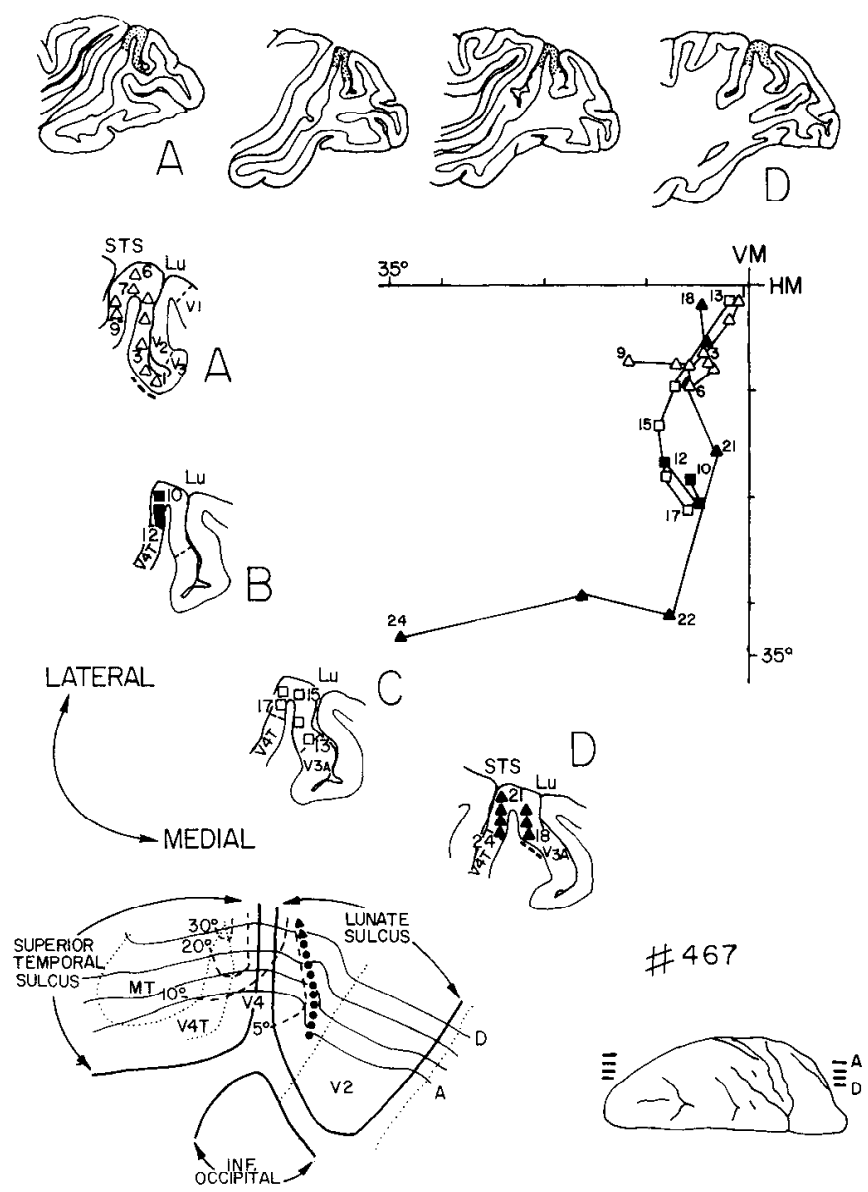

Figure 17. Location of receptive-field centers in dorsal V4 (shaded) recorded at sites indicated in parasagittal sections (A-D) cut at the levels indicated in the dorsal view of the brain (animal 467). Flattened map shows the visual topography of dorsal V4 for this animal. See also legends to Figures 1 and 3.

or the slopes $(t=0.34, p>0.5)$ for dorsal and ventral V4. However, the slope of the function for V3 was significantly different from that for V4 $(t=7.86, p<0.001)$. That is, receptive-field size increased with increasing eccentricity more rapidly in V4 than in V3.

The size of receptive fields in V3 was significantly larger than in V1 $(t=3.4, p<0.001)$, but did not differ significantly from those in V2 $(t=1.9, p>0.05)$.

Previously we found, for both V1 and MT, that the slope of the functions relating field size and eccentricity was slightly larger when obtained with multiunit recordings than with singleunit recordings (Albright and Desimone, 1984). Presumably, therefore, the present curves also slightly overestimate receptive-field size, but equally so for the different regions.

Cortical magnification. The cortical magnification factor, i.e., the distance in millimeters between 2 recording sites (separated by $0.5-3 \mathrm{~mm}$ ) divided by the distance in degrees between the centers of the receptive fields recorded at those sites, decreases with increasing eccentricity in both V3 and V4. Cortical magnification is plotted as a function of the mean of the eccentricities of the pairs of receptive fields for V3d and V3v in Figure $20 \mathrm{~A}$. Best-fitting power functions were obtained with the method of least squares for each set of data. There was no significant or even suggestive difference in either the slope or intercept of these
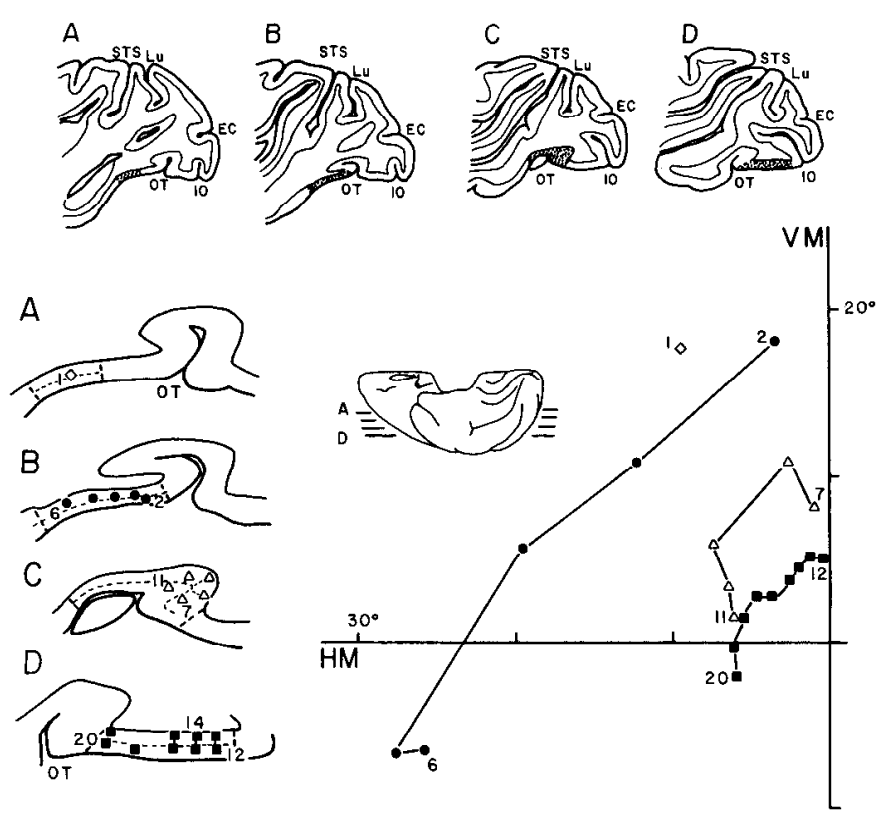

Figure 18. Location of receptive-field centers in ventral V4 (shaded) recorded at the sites indicated in a series of parasagittal sections (A-D) cut at the levels indicated in the ventral view of the brain (animal 467). For abbreviations see Figures 1 and 5.

functions for V3d and V3v (slope, $t=1.05, p>0.2$; intercept, $t=-1.95, p>0.05$ ). The equation for dorsal and ventral V3 combined was $M=1.82 E^{-0.74}$, where $M$ is cortical magnification and $E$ is mean eccentricity of pairs of receptive-field centers. Cortical magnification factor is plotted as a function of mean eccentricity for dorsal and ventral V4 in Figure 20B. Again, there was no difference in either the slope or intercept for dorsal and ventral V4 (slope, $t=1.41, p>0.1$; intercept, $t=1.80$, $p>0.05$ ). The equation for the power function relating cortical magnification and eccentricity for V4 was $M=3.01 E^{-0.90}$. Although there was a tendency for magnification to be greater in the central region of V4 as compared to V3, neither the slope nor the $y$-intercept of the curves for V3 (combining V3d and $\mathrm{V} 3 \mathrm{v}$ ) and V4 (combining dorsal and ventral) was significantly different (slope, $t=1.17, p>0.2 ; y$-intercept, $t=1.88, p>$ $0.05)$.

\section{Myeloarchitecture}

In sections stained for myelin with the Heidenhain-Woelcke method, we were usually able to distinguish V3 and V4 from each other and from most of the surrounding areas (Fig. 21). However, the stain is sometimes variable from section to section. Thus, in a particular section, a given border was sometimes impossible to discern, and we had to use adjacent sections to do so. In general, myeloarchitectonic borders could be determined to within $1 \mathrm{~mm}$, but the uncertainty could be greater when the border fell in a section that was cut tangentially through the relevant cortex. It should be stressed that the myeloarchitectonic descriptions given below apply only to the HeidenhainWoelcke stain and not to the Gallyas stain, although most of these areas also appear differentiable with the Gallyas stain (cf. Van Essen, 1985; Ungerleider and Desimone, 1986a; Colby et al., 1988). In our experience, when the borders of the areas are discernible with both stains, they coincide (Gattass et al., 1986).

Area V3. The myeloarchitectonic pattern of $\mathrm{V} 3$ differs from 

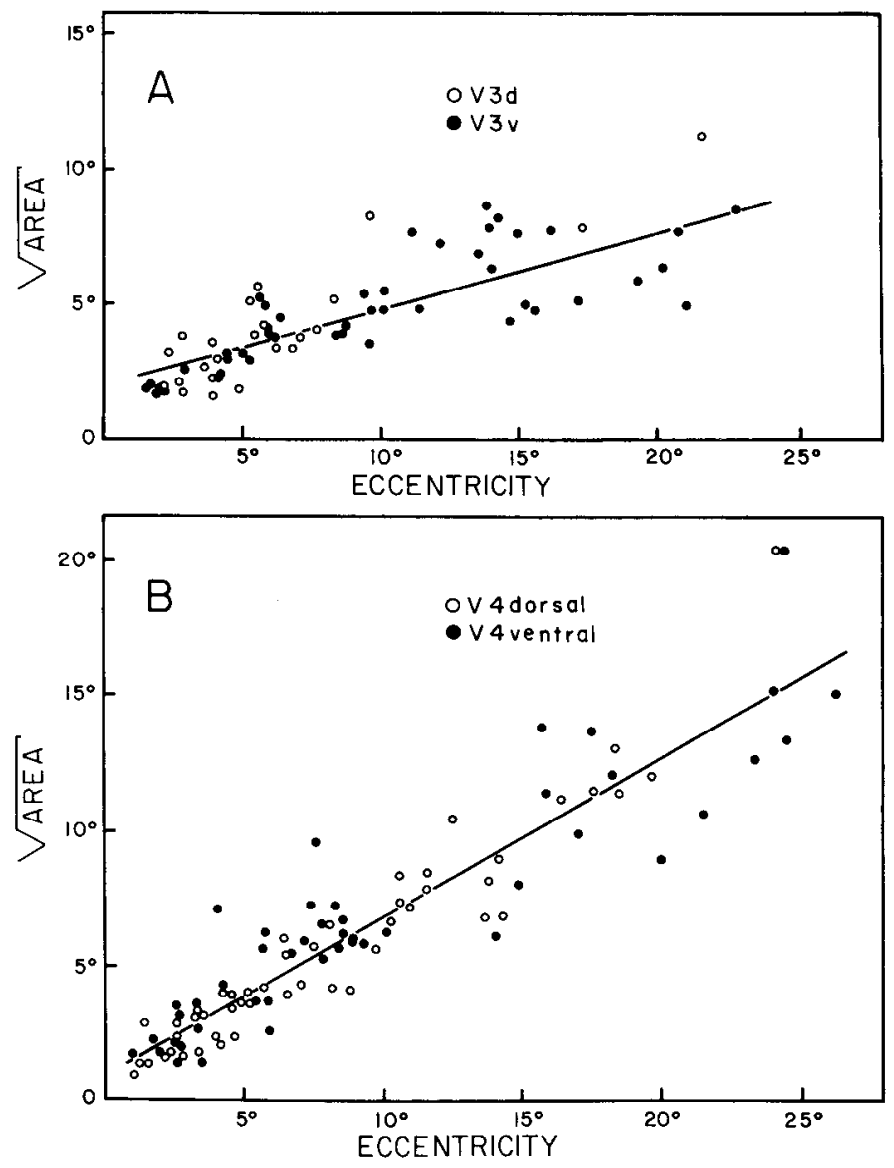

Figure 19. Receptive-field size ( $\sqrt{\text { area }}$ ) as a function of retinal eccentricity for V3 $(A)$ and V4 $(B)$. Data for both areas were obtained from the same animal (482) with the same electrode. The straight lines were fitted by the method of least squares.

that of V2. V2 shows a homogeneous, broad, dark band of fibers that extends from layer VI through layer IV and fades out in the bottom of layer III (Gattass et al., 1981). In V3 this band becomes less homogeneous, more stratified, and the inner band of Baillarger becomes visible. In the region of representation of the center of gaze, however, it was often difficult to distinguish V3 and V2. Area V3 could not be distinguished from V3A with the Heidenhain-Woelcke stain.

Area V4. V4 is distinguishable from V2, V3, and V3A by the presence of much more prominent inner and outer bands of Baillarger. The inner band is thicker than the outer band. The bands are quite distinct because the intermediate region is pale and the region between the white matter and the inner band of Baillarger is also pale.

Bordering V4 anteriorly are areas V4T, TEO, and VF, which differ from V4 in their myeloarchitecture. V4T is similar to V4 except that there is no pale region between the white matter and the inner band of Baillarger. TEO is characterized by an inner band of Baillarger that is broader and paler than that obscrved in V4, and the outer band of Baillarger is thicker and more prominent than that in V4. As in V4T, there is no pale region between white matter and the inner band of Baillarger in TEO. With the Heidenhain-Woelcke stain, VF appears much paler than the adjacent V4, and the inner band of Baillarger that is prominent in V4 is virtually absent in VF.
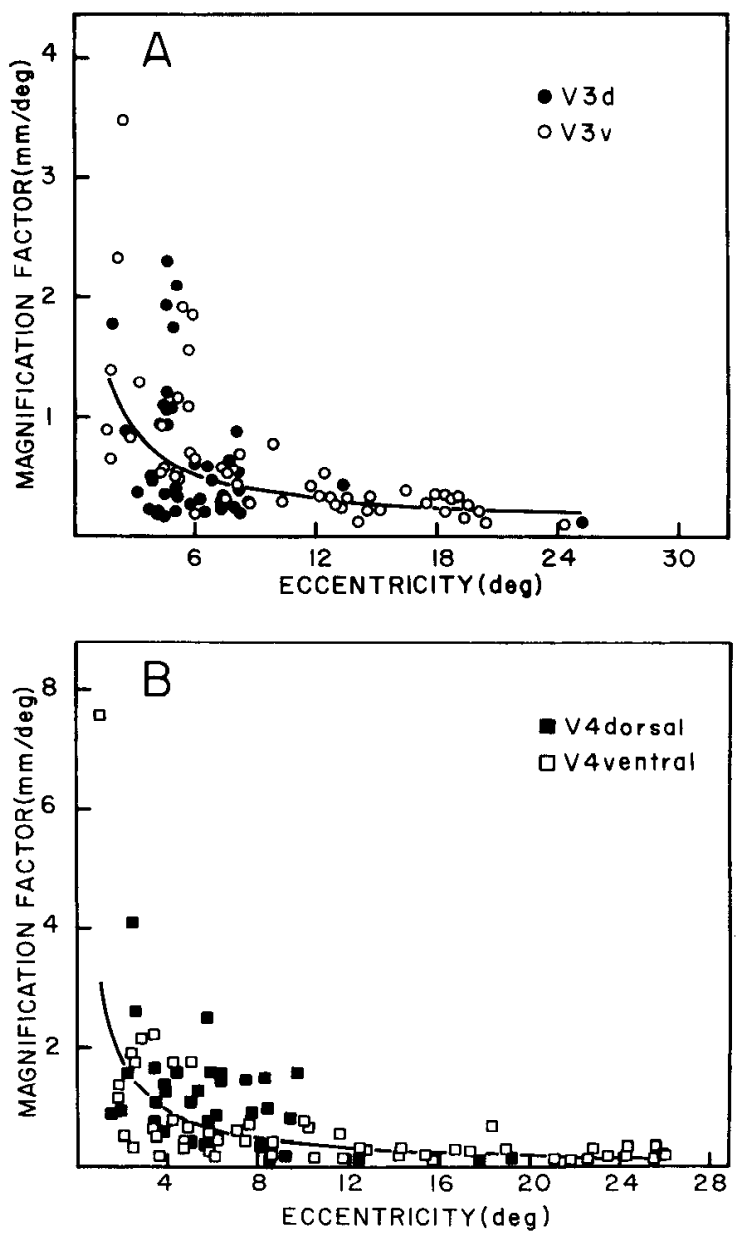

Figure 20. Magnification factor in $\mathrm{mm} / \mathrm{deg}$ as a function of eccentricity for V3 $(A)$ and V4 $(B)$. Data for both areas were obtained from the same animal (482). The power functions were fitted with the method of least squares.

\section{Discussion}

Visual area $V 3$

Anterior to V2, we found a narrow strip of myeloarchitectonically distinguishable cortex in which the lower visual field is represented dorsally, the upper visual field ventrally, and the center of gaze laterally. The posterior border is the representation of the horizontal meridian, and most or all of the anterior border is the representation of the vertical meridian. There is a discontinuity of a few millimeters between the dorsal portion, or $\mathrm{V} 3 \mathrm{~d}$, and the ventral portion, or $\mathrm{V} 3 \mathrm{v}$. We found 2 types of organization along the anterior border of $\mathrm{V} 3 \mathrm{~d}$, as illustrated in Figure 22, middle. In one type, this border is simply the representation of the lower vertical meridian. In the other, at the anterior border the representation of the lower vertical meridian is divided into 2 portions by a representation of a portion of the lower visual field lying between the meridians.

The existence of a strip of visuotopically organized cortex anterior to V2 in the macaque was first proposed by Zeki (1969, 1978). Zeki called this strip V3, and we use this name for the area described here. However, it should be noted that 7eki never published any physiological evidence for the ventral portion of V3. Furthermore, his original anatomical evidence for a ventral V3, namely, a topographic projection from ventral V1, has not been confirmed (Newsome et al., 1980; Van Essen et al., 1982; 


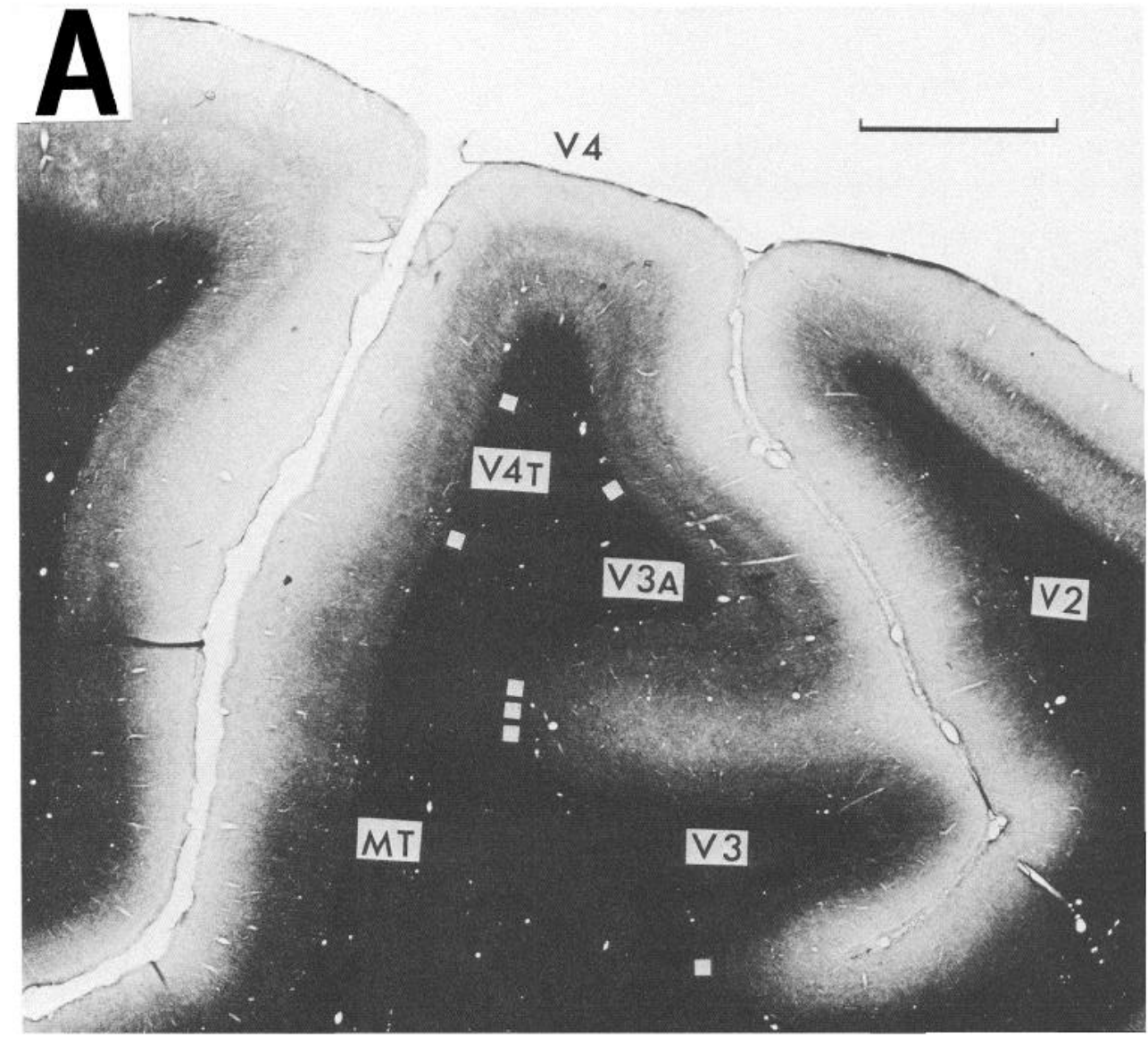

Figure 21. Microphotographs of the dorsal $(A)$ and ventral $(B)$ portions of 3 parasagittal sections stained for myelin (Heidenhain-Woelke method) showing $\mathrm{V} 3, \mathrm{~V} 4$, and neighboring areas (animal 467). Borders (or transition zones) are indicated with squares.
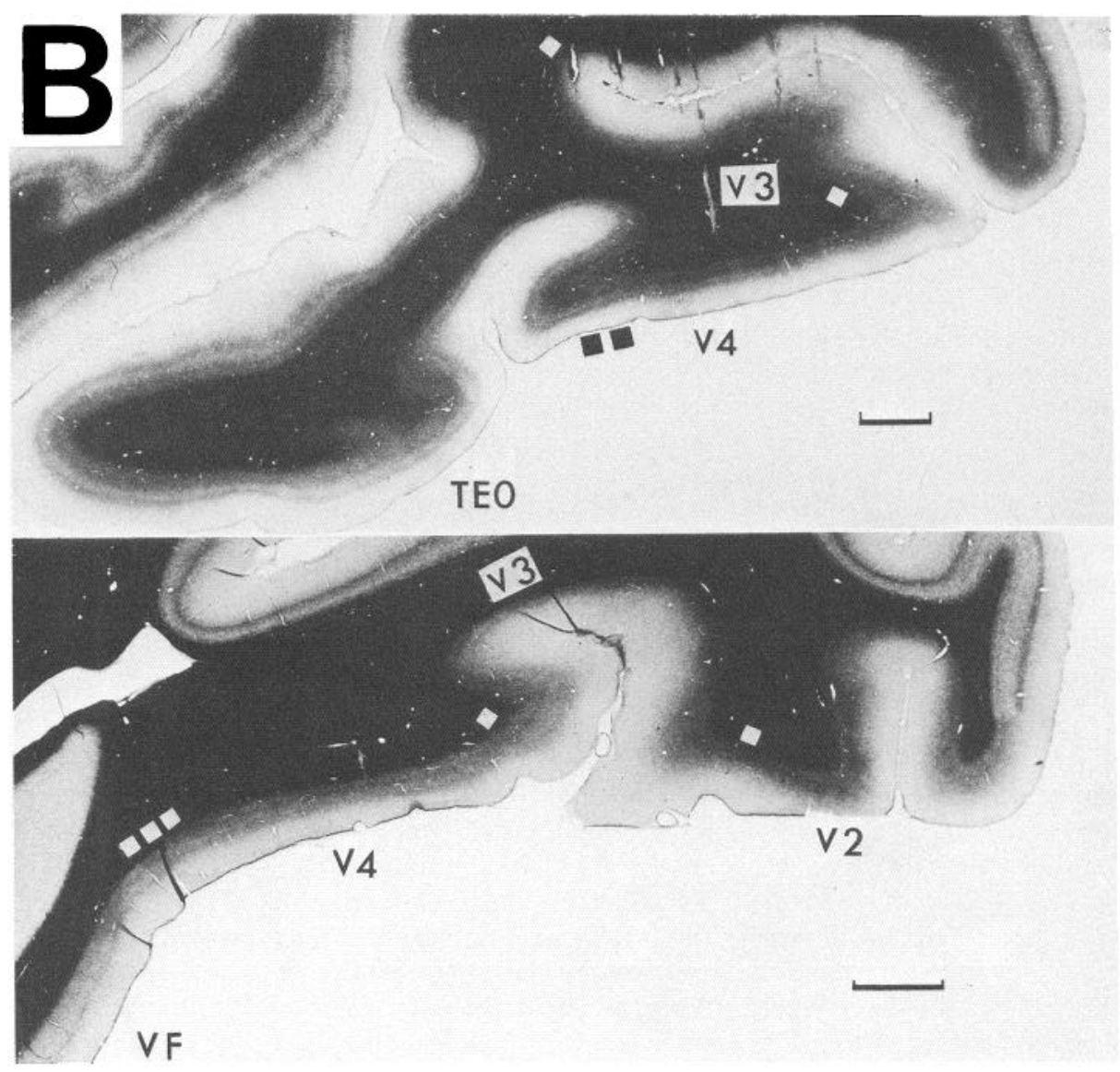
Ungerleider, 1985). Subsequently, however, Gattass et al. (1981) and Van Essen et al. (1982) reported physiological evidence for a visuotopically organized strip of cortex immediately anterior to the ventral portion of $\mathrm{V} 2$. This area was shown to receive a topographically organized projection from V2 (Ungerleider et al., 1983).

Van Essen and his colleagues have pointed out that the dorsal and ventral portions of V3 differ in several ways, including their different afferent and efferent connections, a more variable and irregular visuotopic organization for dorsal V3 than for ventral V3 (which we also found), and more directionally selective and fewer color-selective cells in the dorsal region compared to the ventral one (Burkhalter et al., 1986). They have suggested that these differences be indicated by giving the 2 portions different names-V3 or V3d for the dorsal area, and VP or V3V for the ventral. We have adopted the terms V3d and V3v (as have Ungerleider and Desimone, 1986b), since this terminology recognizes the differences between the 2 regions without entirely replacing the original terminology or implying that the dorsal and ventral portions are different visual areas in the sense that $\mathrm{V} 1, \mathrm{~V} 2, \mathrm{~V} 3$, and V4 are clearly different from one another.

Since we carried out the above studies, Van Essen and his collaborators have reported on the location and visuotopic organization of $\mathrm{V} 3 \mathrm{~d}$ and $\mathrm{V} 3 \mathrm{~V}$ on the basis of studies of myeloarchitecture, the topography of afferent, efferent, and callosal connections, and the location of receptive fields (Burkhalter et al., 1986; Newsome et al., 1986). In all respects, our conclusions on the visual topography of V3d and V3v appear identical to theirs, including those concerning the discontinuity between V3d and V3v and the greater variability of V3d. As to Zeki's (1969) original proposal for $\mathrm{V} 3$, it corresponds closely to the $\mathrm{V} 3 \mathrm{v}$ and to the simpler topographical variant of V $3 \mathrm{~d}$ found in the current study.

\section{Visual area $V 4$}

Anterior to V3, we found a myeloarchitectonically distinguishable area extending across the prelunate and preoccipital gyri that contained a single, and somewhat disorderly, representation of the visual field. Since this area corresponds to the area designated "V4" or "V4 complex" by Zeki (1971) and by Van Essen and Zeki (1978), we used that designation, although all their physiological and most of their anatomical data were only from the dorsal portion of this region.

We found that the posterior border of V4 corresponds to the representation of the vertical meridian and that the representation of the horizontal meridian forms, in part, its anterior border. The upper visual field is represented ventrally, the center of gaze on the lateral surface and the lower visual field dorsally. However, unlike in V3, the small portion of the inferior visual field beyond $5^{\circ}$ is represented on the ventral surface near the anterior border of $\mathrm{V} 4$.

In general, the representation of the visual field in the dorsal portion of V4 is somewhat more disorderly and more variable among animals than in its ventral portion, and both are less consistently organized than V3 or V2. Another peculiarity of the organization of $\mathrm{V} 4$ is that the representation of the center of gaze corresponds to what Palmer et al. (1978) have termed a point-to-line transformation, as opposed to the point-to-point organization found in primate V1. That is, the center of gaze is represented by an elongated strip of cortex rather than by a circular region (Fig. 22, lower). One consequence of this, shown in the flattened maps of Figures 3 and 5 and in Figure 4, is that

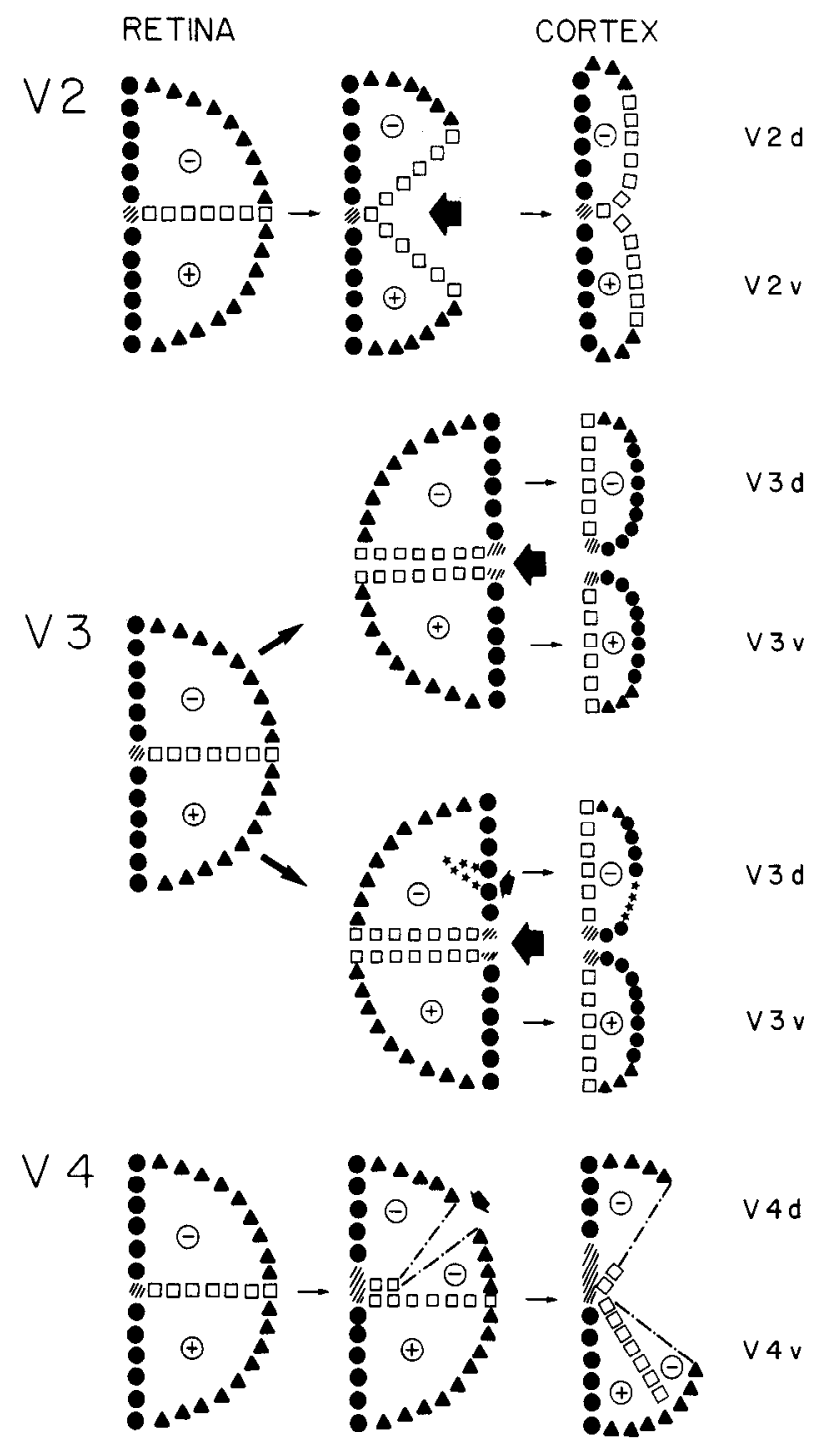

Figure 22. Diagram showing hypothetical transformations (splits) in the representation of the visual field in $\mathrm{V} 2$, in the 2 variants of $\mathrm{V} 3$, and in V4. In V2, and in one variant of V3, the representation is split along the horizontal meridian. In the other variant of V3, there is an additional split in the inferior field. In V4, rather than at the horizontal meridian, the split starts in the lower field along a line oblique to the horizontal meridian and then coincides with the horizontal meridian. Note that all splits take place in the anterior portion of the areas. Symbols as in Figure 3.

the representations of the center of gaze in V4, V3d, V3v, and $\mathrm{V} 2$ are contiguous to each other.

In their study of this region, Van Essen and Zeki (1978) failed to find a systematic representation of the visual field. However, virtually all their recordings were in the central $5^{\circ}-10^{\circ}$ of the visual field. Because of the large size of receptive fields in V4 and the considerable scatter, it is indeed difficult or impossible to discern the organization of V4 if only such a small portion of it is sampled.

In another study of the visuotopic organization of the preIunate gyrus, Maguire and Baizer (1984) reported 2 representations of the inferior quadrant on the prelunate gyrus, separated by a representation of the vertical meridian, in contrast to the single representation found in the present study. One possible reason for the discrepancies between our results and theirs is 
that they studied awake, behaving animals over a long period of time and, thus, were presumably unable to detect all the penetration tracks. Indeed, they state that some penetrations were unmarked and "their positions were reconstructed on the basis of their position in the recording chamber relative to marked tracks and marking pins," a procedure likely to introduce error into the location of the recording sites. This problem, plus the large size and scatter of $\mathrm{V} 4$ receptive fields, the interanimal variability, and the fact that a portion of the inferior visual field is located ventrally rather than dorsally, may account for the discrepancies between our results and theirs. Furthermore, although we found that the organization of dorsal V4 varies from animal to animal (cf. flattened maps in Figs. 3 and 17), we always found a single representation of part of the inferior visual quadrant in dorsal V4. Moreover, of the 3 visuotopic maps illustrated by Maguire and Baizer (1984), in one there is only a single representation of the inferior quadrant (their fig. 5B), and, indeed, it is quite similar to the one found in all our animals. Finally, it should be noted that the pattern of collosal input to the prelunate gyrus described by Van Essen et al. (1982) is fully compatible with our interpretation and quite incompatible with Maguire and Baizer's.

The somewhat "crude" or "disorderly" representation of the visual field in V4 compared to that in V3, V2, or V1 may have been due to one or both of the following factors. The first is the large receptive fields in V4, since both in $\mathrm{V} 1$ and in MT, the scatter in the visuotopic organization is proportional to the receptive-field size (Hubel and Wiesel, 1974; Gattass and Gross, 1981). A second is that V4 receives a variety of different inputs, including from visuotopically organized areas (V2 and V3), a crudely visuotopically organized area (TEO), and a nonvisuotopically organized area (inferior temporal cortex).

\section{References}

Albright, T. D., and R. Desimone (1984) Precision of visuotopic organization of area MT in the macaque. Soc. Neurosci. Abstr. 10:474.

Allman, J. M. (1981) Reconstructing the evolution of the brain in primates through the use of comparative neurophysiological and neuroanate mical data. In Primate Brain Evolution: Methods and Concepts, E. Amstrong, and D. Falk, eds., pp. 13-28, Plenum, New York.

Burkhalter, A., D. J. Felleman, W. T. Newsome, and D. C. Van Essen (1986) Anatomical and physiological asymmetries related to visual areas V3 and VP in the macaque extrastriate cortex. Vision Res. 26: 63-80.

Colby, C. L., R. Gattass, C. R. Olson, and C. G. Gross (1988) Topographic organization of cortical afferents to extrastriate visual area in the macaque: A dual tracer study. J. Comp. Neurol. 269: 392-413.

Covey, E., R. Gattass, and C. G. Gross (1982) A new visual area in the parieto-occipital sulcus of the macaque. Soc. Neurosci. Abstr. 8: 681 .

Daniel, P. M., and D. Whitteridge (1961) The representation of the visual field on the cerebral cortex in monkeys. J. Physiol. (Lond.) 159: 203-221.

Desimone, R., and L. G. Ungerleider (1986) Multiple visual areas in the caudal superior temporal sulcus of the macaque. J. Comp. Neurol. 248: 147-163.

Felleman, D. J., E. A. De Yoe, and D. C. Van Essen (1986) Two topographically organized visual areas in ventral extrastriate cortex of the macaque monkey. Soc. Neurosci. Abstr. 11: 1246.

Fenstemaker, S. B., C. R. Olson, and C. G. Gross (1984) Afferent connections of macaque visual areas V4 and TEO. ARVO Abstr. 25: 213.

Fenstemaker, S. B., T. D. Albright, and C. G. Gross (1985) Organization and neuronal properties of visual area TEO. Soc. Neurosci. Abstr. 11: 1012

Fenstemaker, S. B., A. M. Sauceda, T. D. Albright, and C. G. Gross (1986) A comparison of subcortical afferents to visual cortical areas V4, TEO and MT in the macaque. Soc. Neurosci. Abstr. 12: 1364.
Gattass, R., and C. G. Gross (1981) Visual topography of the striate projection zone in the posterior superior temporal sulcus (MT) of the macaque. J. Neurophysiol. 46: 521-638.

Gattass, R., C. G. Gross, and J. H. Sandell (1981) Visual topography of V2 in the macaque. J. Comp. Neurol. 21: 519-539.

Gattass, R., A. P. B. Sousa, and E. Covey (1985) Cortical visual areas of the macaque: Possible substrates for pattern recognition mechanisms. In Pattern Recognition Mechanisms, C. Chagas, R. Gattass, and C. Gross, eds., pp. 1-20, Pontificae Academiae Scientiarum Scripta Varia No. 54.

Gattass, R., M. Fiorani, Jr., M. G. P. Rosa, and A. P. B. Sousa (1986) Visual areas in the posterior superior temporal sulcus in the Cehus. Soc. Neurosci. Abstr. 12: 1366.

Gross, C. G., C. J. Bruce, R. Desimone, J. Fleming, and R. Gattass (1981) Cortical visual areas of the temporal lobe. In Cortical Sensory Organization, vol. 2. Multiple Visual Areas, C. N. Woolsey, ed., pp. 187-216, Humana, Englewood Cliffs, NJ.

Hubel, D. H., and T. N. Wiesel (1974) Uniformity of monkey striate cortex: A parallel relationship between field size, scatter and magnification factor. J. Comp. Neurol. 158: 295-306.

Iwai, E., and M. Mishkin (1969) Further evidence on the locus of the visual area in the temporal lobe of the monkey. Exp. Neurol. 25: 585594.

Maguire, W. M., and J. S. Baizer (1984) Visuotopic organization of the prelunate gyrus in rhesus monkey. J. Neurosci. 4: 1690-1704.

Maunsell, J. H. R., and D. C. Van Essen (1983) The connections of the middle temporal visual area (MT) and their relationship to a cortical hierarchy in the macaque monkey. J. Neurosci: 3: 2563-2586.

Mishkin, M. (1972) Cortical visual areas and their interactions. In Brain and Human Behavior, A. G. Karczmar and J. C. Eccles, eds., pp. 187-208, Springer-Verlag, Berlin.

Newsome, W. T., and R. H. Wurtz (1982) Identification of architectonic zones containing visual tracking cells in the superior temporal sulcus (STS) of macaque monkeys. Invest. Ophthalmol. Vis. Sci. (Suppl.) 22: 238.

Newsome, W. T., J. H. R. Maunsell, and D. C. Van Essen (1980) Areal boundaries and topographic organization of the ventral posterior area (VP) of the macaque monkey. Soc. Neurosci. Abstr. 6: 579.

Newsome, W. T., J. H. R. Maunsell, and D. C. Van Essen (1986) The ventral posterior visual area of the macaque: Visual topography and areal boundaries. J. Comp. Neurol. 252: 129-153.

Palmer, L. A., A. C. Rosenquist, and R. J. Tusa (1978) The retinotopic organization of lateral suprasylvian visual areas in the cat. J. Comp. Neurol. 177: 237-256.

Schein, S. J., R. T. Marrocco, and F. M. De Monasterio (1982) Is there a high concentration of color-selective cells in area V4 of monkey visual cortex? J. Neurophysiol. 47: 193-213.

Ungerleider, L. G. (1985) The corticocortical pathways for object recognition and spatial perception. In Pattern Recognition Mechanisms, C. Chagas, R. Gattass, and C. Gross, eds., pp. 21-37, Pontificiae Academiae Scientiarum Scripta Varia No. 54.

Ungerleider, L. G., and R. Desimone (1986a) Projection to the superior temporal sulcus from the central and peripheral field representations of V1 and V2. J. Comp. Neurol. 248: 164-189.

Ungerleider, L. G., and R. Desimone (1986b) Cortical connections of visual area MT in the macaque. J. Comp. Neurol. 248: 190-222.

Ungerleider, L. G., R. Gattass, A. P. B. Sousa, and M. Mishkin (1983) Projections of area V2 in the macaque. Soc. Neurosci. Abstr. 9: 152.

Van Essen, D. C. (1985) Functional organization of primate visual cortex. In Cerebral Cortex, vol. 3, E. G. Jones and A. A. Peters, eds., pp. 259-329, Plenum, New York.

Van Essen, D. C., and J. H. R. Maunsell (1980) Two dimensional maps of the cerebral cortex. J. Comp. Neurol. 191: 255-281.

Van Essen, D. C., and S. M. Zeki (1978) The topographic organization of rhesus monkey prestriate cortex. J. Physiol. (Lond.) 277: 193-226.

Van Essen, D. C., J. H. R. Maunsell, and J. L. Bixby (1981) The middle temporal visual area in the macaque: Myeloarchitecture, connections, functional propertics and topographic organization. J. Comp. Neurol. 199: 293-326.

Van Essen, D. C., W. T. Newsome, and J. L. Bixby (1982) The pattern of interhemispheric connections and its relationship to extrastriate visual areas in the macaque monkey. J. Neurosci. 2: 265-283.

Weller, R. E., and J. Kaas (1981) Cortical and subcortical connections of visual cortex in primates. In Cortical Sensory Organization, C. Woolsey, ed., pp. 121-155, Humana, Clifton, NJ. 
Zeki, S. M. (1969) Representation of central visual fields in prestriate cortex. Brain Res. 14: 271-291.

Zeki, S. M. (1971) Cortical projections from two prestriate areas in the monkey. Brain Res. 34: 19-35.

Zeki, S. M. (1977) Colour coding in the superior temporal sulcus of rhesus monkey visual cortex. Proc. R. Soc. Lond. [Biol.] 197: 195223.

Zeki, S. M. (1978) The third visual complex of rhesus monkey prestriate cortex. J. Physiol. (Lond.) 177: 245-272. 